\title{
Characteristics of COVID-19-related Clinical Studies and Factors Affecting the Recruitment and Completeness: a Large Cross-sectional Study
}

\author{
Mingxing Lei ( $\Delta 825979020 @ q q . c o m$ ) \\ Chinese PLA General Hospital \\ Jinglan $\mathbf{L i}$ \\ Chinese PLA General Hospital \\ Houchen LV \\ Chinese PLA General Hospital \\ Feng Lin \\ Chinese PLA General Hospital \\ Licheng Zhang \\ Chinese PLA General Hospital
}

\section{Research}

Keywords: COVID-19, Studies, ClinicalTrials.gov, Recruitment status, Risk factors

Posted Date: August 10th, 2021

DOI: https://doi.org/10.21203/rs.3.rs-763055/v1

License: (c) (1) This work is licensed under a Creative Commons Attribution 4.0 International License. Read Full License 


\section{Abstract}

Background: Coronavirus disease 2019 (COVID-19) has generated an unprecedented clinical research response, but the data about the characteristics of COVID-19-related clinical studies were scarce. The study aimed to describe the characteristics of COVID-19-related clinical studies registered at ClinicalTrials.gov and further identify factors affecting the recruitment and completeness of these studies.

Methods: The study extracted 5,672 studies and included 5,430 studies relating to COVID-19 registered at ClinicalTrials.gov. We presented the characteristics of all included clinical studies. Identification of risk factors for recruitment status was achieved using the multiple logistic regression models, and identification of risk factors for completion time was obtained using the multiple Cox proportional hazards regression models. Subgroup analyses were also performed in the interventional studies.

Results: Of the included studies, only 19.59\% (1064/5430) had completed recruitment, and $55.93 \%$ (3037/5430) were interventional studies. The peak of the number of clinical studies relating to COVID-19 was seven months earlier than the first peak of the number of COVID-19 cases globally. In all included studies, participants only including male $(P=0.02)$, Participants including child $(P=0.01)$, smaller enrollment $(P<0.01)$, and studies not being funded by industry $(P=0.01)$ and the National Institutes of Health $(\mathrm{NIH})(\mathrm{P}<0.01)$, and observational studies $(P<0.01)$ tended to be associated to higher completed recruitment rates. Regarding the interventional studies, Participants including child $(P=0.04)$, smaller enrollment $(P<0.01)$, a crossover intervention model $(P<0.01)$, and primary purpose involving in device feasibility $(P<0.01)$ and treatment $(P=0.03)$ were associated with shorter completion time, while being funded by industry $(P=0.01)$ and $\mathrm{NIH}(P<0.01)$, primary purpose involving in basic science $(P<0.01)$, and biological interventions $(P<0.01)$ were associated with longer completion time.

Conclusion: A multitude of clinical studies relating to COVID-19 are registered in responding to the pandemic and the response is rapid and timely, but these clinical studies are frequently not completed. Increased focus on establishing global initiatives and networks to coordinate recruitment efforts may be needed. Several independent risk factors are identified to guide the design of COVID-19-related clinical studies. This may be significant to avoid waste and ensure that the participation of all participants in clinical researches contributes to the treatment or prevention of COVID-19.

\section{Background}

A recent infection with the new severe acute respiratory syndrome coronavirus (SARS-CoV-2) has been reported in 2019 and has quickly spread worldwide in 2020. This infection, namely coronavirus disease 2019 (COVID-19), was considered a great pandemic in March 2020 by the World Health Organization due to its fast dissemination [1]. It has acclaimed for 183.56 million cases and 3.98 million deaths all over the world [1]. Meanwhile, the pandemic has generated an unprecedented clinical research response in order to find effective approaches curbing COVID-19 [2]. High-quality evidence generated by clinical studies is needed to advance care for patients with COVID-19, those who are susceptible to it, and other patients 
during the pandemic. Although related clinical studies were continuously reported and researchers spent considerable effort on these studies processes, many unforeseen events could occur during the course of a clinical study. Consequently, as reported previously a multitude of studies were often not completed as initially planned, and the proportion of uncompleted study was as high as $25 \%$, or even above $80 \%$ [3-6]. What's more, about $78 \%$ of trials constrained by mandatory reporting did not report results after a year of completion [7] and fewer than half of trials funded by NIH were published in a peer-reviewed journal even within 30 months of trial completion [8]. As for the published results in the high-impact journals, more than $90 \%$ of trials had at least one discordance between the publication-reported results and the ClinicalTrials.gov-reported results [9].

Premature discontinuation and limited dissemination of findings of these clinical studies raise both resources wasting and ethical concerns. Reducing waste has been identified as an important challenge in clinical researches $[10,11]$. Although studies have highlighted frequent recruitment problems in clinical researches [3], few studies have empirically addressed factors influencing recruitment status and completion time among clinical studies. Notably, identification of risk factors for recruitment status and completion time is capable of guiding the design of clinical studies so as to reduce waste of resources, improve study efficiency, and consequently curb the pandemic earlier. Practical strategies aiming at directing the practice of clinical studies relating to COVID-19 are extremely warranted to control the epidemic as soon as possible.

In 2004, the International Committee of Medical Journal Editors (ICMJE) stated that only researches registered in ClinicalTrials.gov before the recruitment of patients would be considered for publication in major journals. There would be legal repercussions if the registries were inaccurate [12]. Thus, at present, most of the clinical researches are registered in ClinicalTrials.gov including COVID-19 [13-15]. Hence, we searched in the ClinicalTrials.gov database and collected all COVID-19-related researches. In this article, we described the characteristics of those clinical studies, identified potential factors affecting recruitment and completion time, and proposed suggestions to guide future clinical research.

\section{Methods}

\section{Data Sources and Registry Search}

We searched ClinicalTrials.gov on 14 May 2021 and obtained the listed clinical studies related to the coronavirus disease (COVID-19) using the link (https://clinicaltrials.gov/ct2/results?cond=COVID-19, available on 16 June 2021). The ClinicalTrials.gov was the largest registry of clinical studies, and it consisted of protocol information for more than 380,000 studies from 210 different nations in June 2021. The Web site was developed as a result of the Food and Drug Administration Modernization Act of 1997 and is maintained by the National Library of Medicine (NLM) at the National Institutes of Health (NIH), which provides people with easy access to information regarding clinical studies on a variety of diseases and conditions. The information of registered clinical studies is provided by study researchers or sponsors, and it is capable of serving as a quality control process before studies are completed and 
results become public available. When and before studies start, their information is generally submitted to the Web site. The status of all registered studies is updated throughout the process of carrying out the studies.

The registration information of all COVID-19-related studies $(n=5672)$ was downloaded and analyzed. Studies were excluded if the study (1) had a status of no longer available, temporarily not available, and withdraw, (2) was an expanded access protocol, (3) registered before January 1, 2020, (4) was not relevant to COVID-19, (5) had start date before January 1, 2020, and (6) had an extreme value of completion time (less than 0 day or more than 20 years).

\section{Basic Characteristics}

We collected the registration information of all COVID-19-related studies, and the registration information included recruitment status, gender, age, enrollment, funder, study type, outcome measures, and completion time. Among interventional studies, we further collected allocation, intervention model, masking, primary purpose, interventions, and clinical phases. We reported and analyzed data for interventional studies and all studies as a whole, respectively.

\section{Definition of Recruitment Status and Completion time}

Recruitment status was classified into two types: completed and others in the study. Completed meant that the study had ended normally, and participants are no longer being examined or treated (that is, the last participant's last visit has occurred). Others included not yet recruiting, recruiting, enrolling by invitation, active but not recruiting, terminated, and suspended. Completion time was defined as the time interval from start date to completion date. Start date was defined as the actual date on which the first participant was enrolled in a clinical study or the "estimated" study start date was the date that the researchers think would be the study start date. Completion date was defined as the date on which the last participant in a clinical study was examined or received an intervention to collect final data for the primary outcome measure. The "estimated" primary completion date was the date that the researchers think would be the primary completion date for the study.

\section{Potential Risk Factors for Predicting Recruitment Status and Completion time}

When analyzing the potential risk factors associating with recruitment status and completion time, all studies relating to COVID-19 were included in the analysis. The potential risk factors included gender (male vs. female vs. both), Participants including child (yes vs. no), Participants including older adult (yes vs. no), enrollment ( $\leq 50$ vs. $>50$ and $\leq 100$ vs. $>100$ and $\leq 300$ vs. $>300$ and $\leq 900$ vs. $>900$ ), funded by industry (yes vs. no), funded by NIH (yes vs. no), study type (interventional vs. observational), outcome measures including ICU stay (yes vs. no), outcome measures including length of hospital stay (yes vs. no), outcome measures including mortality (yes vs. no), outcome measures including survival (yes vs. no). Child was participates with an age from birth to 17 years and older adults meant participates with an age of more than 65 years old. Enrollment is the number of participants in a clinical study. The 
"estimated" enrollment is the target number of participants that the researchers need for the study. The study was funded by industry, for example: pharmaceutical and device companies.

Regarding interventional studies, apart from the above-mentioned potential risk factors, we further collected allocation ( $\mathrm{n} / \mathrm{a}$ vs. non-randomized vs. randomized), intervention model (single vs. sequential vs. parallel vs. factorial vs. crossover), masking (none vs. single vs. double vs. triple vs. quadruple), primary purpose (including basic science (yes vs. no), device feasibility (yes vs. no), diagnostic test (yes vs. no), health services research (yes vs. no), prevention (yes vs. no), screening (yes vs. no), supportive care (yes vs. no), treatment (yes vs. no)), interventions (biological (yes vs. no), behavioral (yes vs. no), device (yes vs. no), diagnostic test (yes vs. no), drug (yes vs. no)), and phases (not applicable vs. phase 1 vs. phase 2 vs. phase 3 vs. phase 4). The studies which were marked as phase 1 and phase 2 were classified into phase 2; the studies which were marked as phase 2 and phase 3 were classified into phase 3 in the study. Allocation refers to a method that used to assign participants to an arm of a clinical study. Intervention models refer to the general design of the strategy for assigning interventions to participants in a clinical study.

\section{Statistical Analyses}

Absolute numbers and percentages for categorical variables and medians and corresponding $95 \%$ interval confidence for continuous variables were presented in the study. The univariate and multivariate analyses of recruitment status were evaluated using logistic regression models, respectively. The univariate and multivariate analyses of completion time were evaluated using the Cox proportional hazards regression models, respectively. The univariate analysis of completion time was also evaluated using the log-rank test. Kaplan-Meier curves for each significant variables associating with completion time were also drawn in the study. A P value of 0.05 or less was considered statistically significant. Statistical analysis was performed using SAS 9.4 software for windows XP (SAS Institute Inc., Cary, NC).

\section{Results}

\section{Basic Characteristics of Studies}

We obtained 5,672 listed clinical studies relating to COVID-19 and a map about the number of clinical studies relating to COVID-19 in the whole world (Fig. 1). The figure indicates that Europe had the largest number of studies relating to COVID-19 $(n=2096)$, followed by America $(n=1304)$. According to the exclusive and inclusive criteria (Fig. 2), 5430 clinical studies were finally included in the analysis. The number of studies relating to COVID-19 increased exponentially from January to April in 2020 after the pandemic breakout and reached its peak in April 2020 with about more than 800 clinical studies registered at ClinicalTrial.gov that month (Fig. 3). After August 2020, the number of clinical studies remained stable, ranging from 200 to 300 .

As for COVID-19 cases, the number rapidly increased from about 2000 cases in January 2020 to about 20 million cases in November 2020. After reaching its peak in November 2020, the number gradually has 
decreased until February 2021 and then went up again. Notably, the peak of the number of clinical studies relating to COVID-19 was seven months earlier than the first peek of the number of COVID-19 cases (Fig. 3).

In the entire studies, $19.59 \%$ (1064/5430) studies had completed recruitment, $96.96 \%$ (5265/5430) studies involved in both male and female participants, $16.65 \%$ (904/5430) studies included child participants, $91.14 \%$ (4949/5430) studies included older adults, and 55.93\% (3037/5430) studies were interventional studies. The majority of studies $(24.73 \%, 1343 / 5430)$ enrolled participants ranging from 100 to 300 , followed by participants less than $50(21.07 \%, 1144 / 5430)$. Studies funded by industry were not common $(19.52 \%, 1060 / 5430)$ and funded by $\mathrm{NIH}$ were rare $(2.76 \%, 150 / 5430)$. The median time from the start date to completion date was 276.00 days ( $95 \% \mathrm{Cl}$ : $266.23-285.77$ days). More details are shown in Table 1. 
Table 1

Characteristics of studies relating to COVID-19.

\section{Characteristics}

Recruitment status

Completed

Others

Gender

Male

Female

Both

Participants including child

Yes

No

Participants including older adult

Yes

No

Enrollment

$\leq 50$

$>50$ and $\leq 100$

$>100$ and $\leq 300$

$>300$ and $\leq 900$

$>900$

Funded by industry

Yes

No

Funded by $\mathrm{NIH}$

Yes

No

Study type

Interventional
Samples $(n=5430)$

$19.59 \%(1064 / 5430)$

$80.41 \%(4366 / 5430)$

$0.77 \%(42 / 5430)$

$2.27 \%(123 / 5430)$

$96.96 \%(5265 / 5430)$

$16.65 \%(904 / 5430)$

$83.35 \%(4526 / 5430)$

$91.14 \%$ (4949/5430)

$8.86 \%(481 / 5430)$

$21.07 \%(1144 / 5430)$

$17.99 \%$ (977/5430)

$24.73 \%(1343 / 5430)$

$16.54 \%$ (898/5430)

19.67\% (1068/5430)

$19.52 \%(1060 / 5430)$

$80.48 \%$ (4370/5430)

$2.76 \%(150 / 5430)$

$97.24 \%(5280 / 5430)$

$55.93 \%(3037 / 5430)$ 


\begin{tabular}{|ll|}
\hline Characteristics & Samples $(\mathbf{n}=\mathbf{5 4 3 0})$ \\
\hline Observational & $44.07 \%(2393 / 5430)$ \\
\hline Outcome measures including ICU stay & $2.60 \%(141 / 5430)$ \\
\hline Yes & $97.40 \%(5289 / 5430)$ \\
\hline Outcome measures including length of hospital stay & \\
\hline Yes & $4.44 \%(241 / 5430)$ \\
\hline No & $95.56 \%(5189 / 5430)$ \\
\hline Outcome measures including mortality & $26.94 \%(1463 / 5430)$ \\
\hline Yes & $73.06 \%(3967 / 5430)$ \\
\hline No & \\
\hline Outcome measures including survival & $3.85 \%(209 / 5430)$ \\
\hline Yes & $96.15 \%(5221 / 5430)$ \\
\hline No & $276.00(266.23-285.77)$ \\
\hline Time interval from start date to completion date (median, 95\% Cl, days) & \\
\hline $\begin{array}{l}\text { Abbreviations: COVID-19, corona virus disease } 2019 ; \mathrm{NIH}, \text { national institutes of health; ICU, intensive } \\
\text { care unit; Cl, confident interval. }\end{array}$ & \\
\hline
\end{tabular}

When subgroup analysis of interventional studies relating to COVID-19, 3037 studies were included in the analysis. The basic information of interventional studies is presented in Table 2. In detail, the multitude of interventional studies were randomized $(73.79 \%, 2241 / 3037)$, parallel intervention model $(71.65 \%$, $2176 / 3037)$, and none masking $(51.76 \%, 1572 / 3037)$. Phases, interventions types, and primary purposes were also shown. 
Table 2

Characteristics of interventional studies relating to COVID-19.

\section{Characteristics}

Recruitment status

Completed

Others

Gender

Male

Female

Both

Participants including child

Yes

No

Participants including older adult

Yes

No

Enrollment

$\leq 50$

$>50$ and $\leq 100$

$>100$ and $\leq 300$

$>300$ and $\leq 900$

$>900$

Funded by industry

Yes

No

Funded by $\mathrm{NIH}$

Yes

No

Outcome measures including ICU stay

Yes
Samples $(n=3037)$

$15.90 \%(483 / 3037)$

$84.10 \%(2554 / 3037)$

$0.95 \%(29 / 3037)$

$1.25 \%(38 / 3037)$

$97.79 \%(2970 / 3037)$

$9.68 \%(294 / 3037)$

$90.32 \%(2743 / 3037)$

$91.37 \%(2775 / 3037)$

$8.63 \%(262 / 3037)$

$27.59 \%(838 / 3037)$

$20.15 \%(612 / 3037)$

$24.79 \%(753 / 3037)$

$14.49 \%(440 / 3037)$

$12.97 \%(394 / 3037)$

$29.31 \%(890 / 3037)$

$70.69 \%(2147 / 3037)$
$2.73 \%(83 / 3037)$

$97.27 \%(2954 / 3037)$ 


\section{Characteristics}

No

Outcome measures including length of hospital stay

Yes

No

Outcome measures including mortality

Yes

No

Outcome measures including survival

Yes

No

Allocation

$\mathrm{N} / \mathrm{A}$

Non-randomized

Randomized

Intervention Model

Single

Sequential

Parallel

Factorial

Crossover

Masking

None

Single

Double

Triple

Quadruple

Primary purpose

Basic science
Samples $(n=3037)$

$96.90 \%(2943 / 3037)$

$5.83 \%(177 / 3037)$

$94.17 \%(2860 / 3037)$

$33.52 \%(1018 / 3037)$

$66.48 \%(2019 / 3037)$

$5.04 \%(153 / 3037)$

$94.96 \%(2884 / 3037)$

$17.25 \%(524 / 3037)$

$8.96 \%(272 / 3037)$

$73.79 \%(2241 / 3037)$

$18.67 \%(567 / 3037)$

$5.30 \%(161 / 3037)$

$71.65 \%(2176 / 3037)$

$1.38 \%(42 / 3037)$

$3.00 \%(91 / 3037)$

$51.76 \%(1572 / 3037)$

$10.80 \%(328 / 3037)$

$13.43 \%(408 / 3037)$

$8.92 \%(271 / 3037)$

$15.08 \%(458 / 3037)$ 


\section{Characteristics}

Yes

No

Device feasibility

Yes

No

Diagnostic test

Yes

No

Health services research

Yes

No

Prevention

Yes

No

Screening

Yes

No

Supportive care

Yes

No

Treatment

Yes

No

Interventions

Biological

Yes

No

Behavioral
Samples $(n=3037)$

$1.15 \%(35 / 3037)$

98.85\% (3002/3037)

$0.26 \%(8 / 3037)$

$99.74 \%(3029 / 3037)$

$4.87 \%(148 / 3037)$

$95.13 \%(2889 / 3037)$

$2.73 \%(83 / 3037)$

$97.27 \%(2954 / 3037)$

$17.45 \%(530 / 3037)$

$82.55 \%(2507 / 3037)$

$1.22 \%(37 / 3037)$

$98.78 \%$ (3000/3037)

4.94\% (150/3037)

$95.06 \%(2887 / 3037)$

$62.03 \%(1884 / 3037)$

$37.97 \%(1153 / 3037)$
$17.16 \%(521 / 3037)$

$82.84 \%(2516 / 3037)$ 


\section{Characteristics}

Samples $(n=3037)$

Yes

$8.26 \%(251 / 3037)$

No

$91.74 \%(2786 / 3037)$

Device

Yes

6.55\% (199/3037)

No

$93.45 \%(2838 / 3037)$

Diagnostic test

Yes

$4.02 \%(122 / 3037)$

No

95.98\% (2915/3037)

Drug

Yes

$45.11 \%$ (1370/3037)

No

$54.89 \%(1667 / 3037)$

Phases

Not applicable

$37.64 \%(1143 / 3037)$

Phase 1

$8.69 \%(264 / 3037)$

Phase 2

$27.89 \%(847 / 3037)$

Phase 3

$20.74 \%(630 / 3037)$

Phase 4

$5.04 \%(153 / 3037)$

Time interval from start date to completion date (median, 95\% Cl, days)

$276.00(264.43-287.57)$

Abbreviations: COVID-19, corona virus disease 2019; NIH, national institutes of health; ICU, intensive care unit; $\mathrm{Cl}$, confident interval.

\section{Risk Factors for Recruitment Status}

According to the multivariate analysis in the entire studies (Table 3), gender ( $\mathrm{OR}=0.74,95 \% \mathrm{Cl}$ : 0.57-0.96, $P=0.02)$, Participants including child $(O R=1.26,95 \% \mathrm{Cl}: 1.05-1.50, P=0.01)$, enrollment $(O R=0.88$, $95 \% \mathrm{Cl}: 0.84-0.93, \mathrm{P}<0.01)$, funded by industry $(\mathrm{OR}=0.78,95 \% \mathrm{Cl}: 0.64-0.94, \mathrm{P}=0.01)$, funded by $\mathrm{NIH}$ $(\mathrm{OR}=0.37,95 \% \mathrm{Cl}: 0.20-0.67, \mathrm{P}<0.01)$, and study type $(\mathrm{OR}=0.59,95 \% \mathrm{Cl}: 0.51-0.68, \mathrm{P}<0.01)$ were found to be significantly associated with recruitment status. More explicitly, participants only including male, Participants including child, smaller enrollment, not being funded by industry and $\mathrm{NIH}$, and observational studies were tend to be relevant to higher completed recruitment rates. 
Table 3

Univariate and multivariate analyses of characteristics for recruitment status in studies relating to COVID19.

\begin{tabular}{|c|c|c|c|c|c|}
\hline \multirow[t]{2}{*}{ Characteristics } & \multirow[t]{2}{*}{$\begin{array}{l}\text { Completed } \\
\text { recruitment rates }\end{array}$} & \multicolumn{2}{|c|}{$\begin{array}{l}\text { Simple logistic } \\
\text { regression }\end{array}$} & \multicolumn{2}{|c|}{$\begin{array}{l}\text { Multiple logistic } \\
\text { regression }\end{array}$} \\
\hline & & $\begin{array}{l}\mathrm{OR}(95 \% \\
\mathrm{Cl})\end{array}$ & $\mathbf{P}$ & $\begin{array}{l}\mathrm{OR}(95 \% \\
\mathrm{Cl})\end{array}$ & $\mathbf{P}$ \\
\hline \multicolumn{6}{|l|}{ Gender } \\
\hline Male & $35.71 \%(15 / 42)$ & \multirow{3}{*}{$\begin{array}{l}0.70 \\
(0.54- \\
0.91)\end{array}$} & \multirow{3}{*}{$\begin{array}{l}<.01 \\
0.01\end{array}$} & \multirow{3}{*}{$\begin{array}{l}0.74 \\
(0.57- \\
0.96)\end{array}$} & \multirow[t]{3}{*}{0.02} \\
\hline Female & $23.58 \%(29 / 123)$ & & & & \\
\hline Both & $\begin{array}{l}19.37 \% \\
(1020 / 5265)\end{array}$ & & & & \\
\hline \multicolumn{6}{|c|}{ Participants including child } \\
\hline Yes & $23.89 \%(216 / 904)$ & \multirow{2}{*}{$\begin{array}{l}1.36 \\
(1.15- \\
1.61)\end{array}$} & \multirow{2}{*}{$\dot{0} 01$} & \multirow{2}{*}{$\begin{array}{l}1.26 \\
(1.05- \\
1.50)\end{array}$} & \multirow[t]{2}{*}{0.01} \\
\hline No & $\begin{array}{l}18.74 \% \\
(848 / 4526)\end{array}$ & & & & \\
\hline \multicolumn{6}{|c|}{ Participants including older adults } \\
\hline Yes & $\begin{array}{l}19.14 \% \\
(947 / 4949)\end{array}$ & \multirow{2}{*}{$\begin{array}{l}0.74 \\
(0.59- \\
0.92)\end{array}$} & \multirow[t]{2}{*}{$<}$. & \multirow{2}{*}{\multicolumn{2}{|c|}{ Insignificance }} \\
\hline No & $24.32 \%(117 / 481)$ & & & & \\
\hline \multicolumn{6}{|l|}{ Enrollment } \\
\hline$\leq 50$ & $\begin{array}{l}22.73 \% \\
(260 / 1144)\end{array}$ & \multirow{5}{*}{$\begin{array}{l}0.93 \\
(0.89- \\
0.97)\end{array}$} & \multirow[t]{5}{*}{$\begin{array}{l}< \\
0.01\end{array}$} & \multirow{5}{*}{$\begin{array}{l}0.88 \\
(0.84- \\
0.93)\end{array}$} & \multirow[t]{5}{*}{$\begin{array}{l}< \\
0.01\end{array}$} \\
\hline$>50$ and $\leq 100$ & $17.40 \%(170 / 977)$ & & & & \\
\hline$>100$ and $\leq 300$ & $\begin{array}{l}21.15 \% \\
(284 / 1343)\end{array}$ & & & & \\
\hline$>300$ and $\leq 900$ & $20.04 \%(180 / 898)$ & & & & \\
\hline$>900$ & $\begin{array}{l}15.92 \% \\
(170 / 1068)\end{array}$ & & & & \\
\hline \multicolumn{6}{|c|}{ Funded by industry } \\
\hline Yes & $\begin{array}{l}14.62 \% \\
(155 / 1060)\end{array}$ & \multirow[t]{2}{*}{$\begin{array}{l}0.65 \\
(0.54- \\
0.79)\end{array}$} & \multirow[t]{2}{*}{$\begin{array}{l}< \\
0.01\end{array}$} & \multirow[t]{2}{*}{$\begin{array}{l}0.78 \\
(0.64- \\
0.94)\end{array}$} & \multirow[t]{2}{*}{0.01} \\
\hline No & $\begin{array}{l}20.80 \% \\
(909 / 4370)\end{array}$ & & & & \\
\hline Funded by NIH & & & & & \\
\hline
\end{tabular}




\begin{tabular}{|c|c|c|c|c|c|}
\hline \multirow[t]{2}{*}{ Characteristics } & \multirow[t]{2}{*}{$\begin{array}{l}\text { Completed } \\
\text { recruitment rates }\end{array}$} & \multicolumn{2}{|c|}{$\begin{array}{l}\text { Simple logistic } \\
\text { regression }\end{array}$} & \multicolumn{2}{|c|}{$\begin{array}{l}\text { Multiple logistic } \\
\text { regression }\end{array}$} \\
\hline & & $\begin{array}{l}\mathrm{OR}(95 \% \\
\mathrm{Cl})\end{array}$ & $\mathbf{P}$ & $\begin{array}{l}\mathrm{OR}(95 \% \\
\mathrm{Cl})\end{array}$ & $\mathbf{P}$ \\
\hline Yes & $8.00 \%(12 / 150)$ & \multirow{2}{*}{$\begin{array}{l}0.35 \\
(0.19- \\
0.63)\end{array}$} & \multirow{2}{*}{$<0}$. & \multirow{2}{*}{$\begin{array}{l}0.37 \\
(0.20- \\
0.67)\end{array}$} & \multirow{2}{*}{$<}$. \\
\hline No & $\begin{array}{l}19.92 \% \\
(1052 / 5280)\end{array}$ & & & & \\
\hline \multicolumn{6}{|l|}{ Study type } \\
\hline Interventional & $\begin{array}{l}15.90 \% \\
(483 / 3037)\end{array}$ & \multirow{2}{*}{$\begin{array}{l}0.59 \\
(0.52- \\
0.68)\end{array}$} & \multirow[t]{2}{*}{$<.01$} & \multirow{2}{*}{$\begin{array}{l}0.59 \\
(0.51- \\
0.68)\end{array}$} & \multirow[t]{2}{*}{$\begin{array}{l}< \\
0.01\end{array}$} \\
\hline Observational & $\begin{array}{l}24.28 \% \\
(581 / 2393)\end{array}$ & & & & \\
\hline \multicolumn{6}{|c|}{$\begin{array}{l}\text { Outcome measures including ICU } \\
\text { stay }\end{array}$} \\
\hline Yes & $20.57 \%(29 / 141)$ & \multirow{2}{*}{$\begin{array}{l}1.06 \\
(0.70- \\
1.61)\end{array}$} & \multirow[t]{2}{*}{0.77} & \multirow{2}{*}{\multicolumn{2}{|c|}{ Insignificance }} \\
\hline No & $\begin{array}{l}19.57 \% \\
(1035 / 5289)\end{array}$ & & & & \\
\hline \multicolumn{6}{|c|}{$\begin{array}{l}\text { Outcome measures including } \\
\text { length of hospital stay }\end{array}$} \\
\hline Yes & $21.99 \%(53 / 241)$ & \multirow{2}{*}{$\begin{array}{l}1.17 \\
(0.85- \\
1.59)\end{array}$} & \multirow[t]{2}{*}{0.34} & \multirow{2}{*}{\multicolumn{2}{|c|}{ Insignificance }} \\
\hline No & $\begin{array}{l}19.48 \% \\
(1011 / 5189)\end{array}$ & & & & \\
\hline \multicolumn{6}{|c|}{$\begin{array}{l}\text { Outcome measures including } \\
\text { mortality }\end{array}$} \\
\hline Yes & $\begin{array}{l}18.39 \% \\
(269 / 1463)\end{array}$ & \multirow{2}{*}{$\begin{array}{l}0.90 \\
(0.77- \\
1.05)\end{array}$} & \multirow[t]{2}{*}{0.17} & \multirow{2}{*}{\multicolumn{2}{|c|}{ Insignificance }} \\
\hline No & $\begin{array}{l}20.04 \% \\
(795 / 3967)\end{array}$ & & & & \\
\hline \multicolumn{6}{|c|}{$\begin{array}{l}\text { Outcome measures including } \\
\text { survival }\end{array}$} \\
\hline Yes & $15.79 \%(33 / 209)$ & \multirow{2}{*}{$\begin{array}{l}0.76 \\
(0.52- \\
1.11)\end{array}$} & \multirow[t]{2}{*}{0.16} & \multirow{2}{*}{\multicolumn{2}{|c|}{ Insignificance }} \\
\hline No & $\begin{array}{l}19.75 \% \\
(1031 / 5221)\end{array}$ & & & & \\
\hline
\end{tabular}


Regarding subgroup analysis of potential risk factors for interventional studies (Table 4), enrollment (OR $=0.88,95 \% \mathrm{Cl}: 0.81-0.94, \mathrm{P}<0.01)$, masking $(\mathrm{OR}=0.91,95 \% \mathrm{Cl}: 0.84-0.97, \mathrm{P}<0.01)$, diagnostic test $(\mathrm{OR}=$ $1.60,95 \% \mathrm{Cl}: 1.08-2.38, \mathrm{P}=0.02)$, and biological interventions $(\mathrm{OR}=0.53,95 \% \mathrm{Cl}: 0.39-0.73, \mathrm{P}<0.01)$ were found to be significantly associated with recruitment status. In detail, smaller enrollment and primary purpose involving in diagnostic test were associated with higher completed recruitment rates, while masking and biological interventions were associated with lower completed recruitment rates. 
Table 4

Univariate and multivariate analyses of characteristics for recruitment status in interventional studies relating to COVID-19.

\begin{tabular}{|c|c|c|c|c|c|}
\hline \multirow[t]{2}{*}{ Characteristics } & \multirow[t]{2}{*}{$\begin{array}{l}\text { Completed } \\
\text { recruitment rates }\end{array}$} & \multicolumn{2}{|c|}{$\begin{array}{l}\text { Simple logistic } \\
\text { regression }\end{array}$} & \multicolumn{2}{|c|}{$\begin{array}{l}\text { Multiple logistic } \\
\text { regression }\end{array}$} \\
\hline & & $\begin{array}{l}\mathrm{OR}(95 \% \\
\mathrm{Cl})\end{array}$ & $\mathbf{P}$ & $\begin{array}{l}\mathrm{OR}(95 \% \\
\mathrm{Cl})\end{array}$ & $\mathbf{P}$ \\
\hline \multicolumn{6}{|l|}{ Gender } \\
\hline Male & $37.93 \%(11 / 29)$ & \multirow{3}{*}{$\begin{array}{l}0.63 \\
(0.45- \\
0.90)\end{array}$} & \multirow[t]{3}{*}{0.01} & \multirow{3}{*}{\multicolumn{2}{|c|}{ Insignificance }} \\
\hline Female & $13.16 \%(5 / 38)$ & & & & \\
\hline Both & $\begin{array}{l}15.72 \% \\
(467 / 2970)\end{array}$ & & & & \\
\hline \multicolumn{6}{|c|}{ Participants including child } \\
\hline Yes & $20.07 \%(59 / 294)$ & \multirow{2}{*}{$\begin{array}{l}1.37 \\
(1.01- \\
1.86)\end{array}$} & \multirow[t]{2}{*}{0.04} & \multirow{2}{*}{\multicolumn{2}{|c|}{ Insignificance }} \\
\hline No & $\begin{array}{l}15.46 \% \\
(424 / 2743)\end{array}$ & & & & \\
\hline \multicolumn{6}{|c|}{ Participants including older adults } \\
\hline Yes & $\begin{array}{l}15.78 \% \\
(438 / 2775)\end{array}$ & \multirow{2}{*}{$\begin{array}{l}0.90 \\
(0.65- \\
1.27)\end{array}$} & \multirow[t]{2}{*}{0.56} & \multirow{2}{*}{\multicolumn{2}{|c|}{ Insignificance }} \\
\hline No & $17.18 \%(45 / 262)$ & & & & \\
\hline \multicolumn{6}{|l|}{ Enrollment } \\
\hline$\leq 50$ & $20.05 \%(168 / 838)$ & \multirow{5}{*}{$\begin{array}{l}0.86 \\
(0.80- \\
0.93)\end{array}$} & \multirow{5}{*}{$\begin{array}{l}< \\
0.01\end{array}$} & \multirow{5}{*}{$\begin{array}{l}0.88 \\
(0.81- \\
0.94)\end{array}$} & \multirow{5}{*}{$\begin{array}{l}< \\
0.01\end{array}$} \\
\hline$>50$ and $\leq 100$ & $13.40 \%(82 / 612)$ & & & & \\
\hline$>100$ and $\leq 300$ & $17.53 \%(132 / 753)$ & & & & \\
\hline$>300$ and $\leq 900$ & $13.41 \%(59 / 440)$ & & & & \\
\hline$>900$ & $10.66 \%(42 / 394)$ & & & & \\
\hline \multicolumn{6}{|c|}{ Funded by industry } \\
\hline Yes & $14.38 \%(128 / 890)$ & $\begin{array}{l}0.85 \\
(0.68- \\
1.06)\end{array}$ & 0.14 & \multirow{3}{*}{\multicolumn{2}{|c|}{ Insignificance }} \\
\hline No & $\begin{array}{l}16.53 \% \\
(355 / 2147)\end{array}$ & & & & \\
\hline Funded by NIH & & & & & \\
\hline
\end{tabular}




\begin{tabular}{|c|c|c|c|c|}
\hline \multirow[t]{2}{*}{ Characteristics } & \multirow[t]{2}{*}{$\begin{array}{l}\text { Completed } \\
\text { recruitment rates }\end{array}$} & \multicolumn{2}{|c|}{$\begin{array}{l}\text { Simple logistic } \\
\text { regression }\end{array}$} & $\begin{array}{l}\text { Multiple logistic } \\
\text { regression }\end{array}$ \\
\hline & & $\begin{array}{l}\mathrm{OR}(95 \% \\
\mathrm{Cl})\end{array}$ & $\mathbf{P}$ & $\begin{array}{l}\mathrm{OR}(95 \% \\
\mathrm{Cl})\end{array}$ \\
\hline Yes & $10.84 \%(9 / 83)$ & \multirow{2}{*}{$\begin{array}{l}0.64 \\
(0.32- \\
1.28)\end{array}$} & \multirow[t]{2}{*}{0.20} & \multirow[t]{2}{*}{ Insignificance } \\
\hline No & $\begin{array}{l}16.05 \% \\
(474 / 2954)\end{array}$ & & & \\
\hline \multicolumn{5}{|c|}{$\begin{array}{l}\text { Outcome measures including ICU } \\
\text { stay }\end{array}$} \\
\hline Yes & $17.02 \%(16 / 94)$ & \multirow{2}{*}{$\begin{array}{l}1.09 \\
(0.63- \\
1.88)\end{array}$} & \multirow[t]{2}{*}{0.76} & \multirow[t]{2}{*}{ Insignificance } \\
\hline No & $\begin{array}{l}15.87 \% \\
(467 / 2943)\end{array}$ & & & \\
\hline \multicolumn{5}{|c|}{$\begin{array}{l}\text { Outcome measures including } \\
\text { length of hospital stay }\end{array}$} \\
\hline Yes & $16.38 \%(29 / 177)$ & \multirow{2}{*}{$\begin{array}{l}1.04 \\
(0.69- \\
1.57)\end{array}$} & \multirow[t]{2}{*}{0.86} & \multirow[t]{2}{*}{ Insignificance } \\
\hline No & $\begin{array}{l}15.87 \% \\
(454 / 2860)\end{array}$ & & & \\
\hline \multicolumn{5}{|c|}{$\begin{array}{l}\text { Outcome measures including } \\
\text { mortality }\end{array}$} \\
\hline Yes & $\begin{array}{l}14.64 \% \\
(149 / 1018)\end{array}$ & \multirow{2}{*}{$\begin{array}{l}0.87 \\
(0.70- \\
1.07)\end{array}$} & \multirow[t]{2}{*}{0.18} & \multirow[t]{2}{*}{ Insignificance } \\
\hline No & $\begin{array}{l}16.54 \% \\
(334 / 2019)\end{array}$ & & & \\
\hline \multicolumn{5}{|c|}{$\begin{array}{l}\text { Outcome measures including } \\
\text { survival }\end{array}$} \\
\hline Yes & $15.69 \%(24 / 153)$ & \multirow{2}{*}{$\begin{array}{l}0.98 \\
(0.63- \\
1.54)\end{array}$} & \multirow[t]{2}{*}{0.94} & \multirow[t]{2}{*}{ Insignificance } \\
\hline No & $\begin{array}{l}15.92 \% \\
(459 / 2884)\end{array}$ & & & \\
\hline \multicolumn{5}{|l|}{ Allocation } \\
\hline N/A & $18.89 \%(99 / 524)$ & \multirow{3}{*}{$\begin{array}{l}0.86 \\
(0.76- \\
0.97)\end{array}$} & \multirow[t]{3}{*}{0.01} & \multirow[t]{3}{*}{ Insignificance } \\
\hline Non-randomized & $18.75 \%(51 / 272)$ & & & \\
\hline Randomized & $\begin{array}{l}14.86 \% \\
(333 / 2241)\end{array}$ & & & \\
\hline \multicolumn{5}{|c|}{ Intervention Model } \\
\hline Single & $19.40 \%(110 / 567)$ & \multirow{2}{*}{$\begin{array}{l}0.98 \\
(0.88- \\
1.09)\end{array}$} & \multirow[t]{2}{*}{0.67} & \multirow[t]{2}{*}{ Insignificance } \\
\hline Sequential & $8.07 \%(13 / 161)$ & & & \\
\hline
\end{tabular}




\begin{tabular}{|c|c|c|c|c|c|}
\hline \multirow[t]{2}{*}{ Characteristics } & \multirow[t]{2}{*}{$\begin{array}{l}\text { Completed } \\
\text { recruitment rates }\end{array}$} & \multicolumn{2}{|c|}{$\begin{array}{l}\text { Simple logistic } \\
\text { regression }\end{array}$} & \multicolumn{2}{|c|}{$\begin{array}{l}\text { Multiple logistic } \\
\text { regression }\end{array}$} \\
\hline & & $\begin{array}{l}\mathrm{OR}(95 \% \\
\mathrm{Cl})\end{array}$ & $\mathbf{P}$ & $\begin{array}{l}\mathrm{OR}(95 \% \\
\mathrm{Cl})\end{array}$ & $\mathbf{P}$ \\
\hline Parallel & $\begin{array}{l}15.07 \% \\
(328 / 2176)\end{array}$ & & & & \\
\hline Factorial & $21.43 \%(9 / 42)$ & & & & \\
\hline Crossover & $25.27 \%(23 / 91)$ & & & & \\
\hline \multicolumn{6}{|l|}{ Masking } \\
\hline None & $\begin{array}{l}17.56 \% \\
(276 / 1572)\end{array}$ & \multirow{5}{*}{$\begin{array}{l}0.87 \\
(0.81- \\
0.93)\end{array}$} & \multirow[t]{5}{*}{$\begin{array}{l}< \\
0.01\end{array}$} & \multirow{5}{*}{$\begin{array}{l}0.91 \\
(0.84- \\
0.97)\end{array}$} & \multirow[t]{5}{*}{$\begin{array}{l}< \\
0.01\end{array}$} \\
\hline Single & $18.90 \%(62 / 328)$ & & & & \\
\hline Double & $15.93 \%(65 / 408)$ & & & & \\
\hline Triple & $11.07 \%(30 / 271)$ & & & & \\
\hline Quadruple & $10.92 \%(50 / 458)$ & & & & \\
\hline \multicolumn{6}{|l|}{ Primary purpose } \\
\hline \multicolumn{6}{|l|}{ Basic science } \\
\hline Yes & $8.57 \%(3 / 35)$ & \multirow{2}{*}{$\begin{array}{l}0.49 \\
(0.15- \\
1.62)\end{array}$} & \multirow[t]{2}{*}{0.24} & \multirow{2}{*}{\multicolumn{2}{|c|}{ Insignificance }} \\
\hline No & $\begin{array}{l}15.99 \% \\
(480 / 3002)\end{array}$ & & & & \\
\hline \multicolumn{6}{|l|}{ Device feasibility } \\
\hline Yes & $25.00 \%(2 / 8)$ & \multirow{2}{*}{$\begin{array}{l}1.77 \\
(0.36- \\
8.79)\end{array}$} & \multirow[t]{2}{*}{0.48} & \multirow{2}{*}{\multicolumn{2}{|c|}{ Insignificance }} \\
\hline No & $\begin{array}{l}15.88 \% \\
(481 / 3029)\end{array}$ & & & & \\
\hline \multicolumn{6}{|l|}{ Diagnostic test } \\
\hline Yes & $25.00 \%(37 / 148)$ & \multirow{2}{*}{$\begin{array}{l}1.83 \\
(1.24- \\
2.69)\end{array}$} & \multirow{2}{*}{ 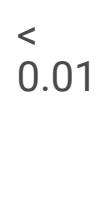 } & \multirow{2}{*}{$\begin{array}{l}1.60 \\
(1.08- \\
2.38)\end{array}$} & \multirow[t]{2}{*}{0.02} \\
\hline No & $\begin{array}{l}15.44 \% \\
(446 / 2889)\end{array}$ & & & & \\
\hline \multicolumn{6}{|c|}{ Health services research } \\
\hline Yes & $14.46 \%(12 / 83)$ & \multirow{2}{*}{$\begin{array}{l}0.89 \\
(0.48- \\
1.66)\end{array}$} & \multirow[t]{2}{*}{0.72} & \multirow{2}{*}{\multicolumn{2}{|c|}{ Insignificance }} \\
\hline No & $\begin{array}{l}15.94 \% \\
(471 / 2954)\end{array}$ & & & & \\
\hline
\end{tabular}




\begin{tabular}{|c|c|c|c|c|c|}
\hline \multirow[t]{2}{*}{ Characteristics } & \multirow[t]{2}{*}{$\begin{array}{l}\text { Completed } \\
\text { recruitment rates }\end{array}$} & \multicolumn{2}{|c|}{$\begin{array}{l}\text { Simple logistic } \\
\text { regression }\end{array}$} & \multicolumn{2}{|c|}{$\begin{array}{l}\text { Multiple logistic } \\
\text { regression }\end{array}$} \\
\hline & & $\begin{array}{l}\mathrm{OR}(95 \% \\
\mathrm{Cl})\end{array}$ & $\mathbf{P}$ & $\begin{array}{l}\mathrm{OR}(95 \% \\
\mathrm{Cl})\end{array}$ & $\mathbf{P}$ \\
\hline Yes & $10.38 \%(55 / 530)$ & \multirow{2}{*}{$\begin{array}{l}0.56 \\
(0.42- \\
0.76)\end{array}$} & \multirow{2}{*}{$<0.01$} & \multirow{2}{*}{\multicolumn{2}{|c|}{ Insignificance }} \\
\hline No & $\begin{array}{l}17.07 \% \\
(428 / 2507)\end{array}$ & & & & \\
\hline \multicolumn{6}{|l|}{ Screening } \\
\hline Yes & $21.62 \%(8 / 37)$ & \multirow{2}{*}{$\begin{array}{l}1.47 \\
(0.68- \\
3.23)\end{array}$} & \multirow[t]{2}{*}{0.34} & \multirow{2}{*}{\multicolumn{2}{|c|}{ Insignificance }} \\
\hline No & $\begin{array}{l}15.83 \% \\
(475 / 3000)\end{array}$ & & & & \\
\hline \multicolumn{6}{|l|}{ Supportive care } \\
\hline Yes & $22.67 \%(34 / 150)$ & \multirow{2}{*}{$\begin{array}{l}1.59 \\
(1.07- \\
2.36)\end{array}$} & \multirow[t]{2}{*}{0.02} & \multirow{2}{*}{\multicolumn{2}{|c|}{ Insignificance }} \\
\hline No & $\begin{array}{l}15.55 \% \\
(449 / 2887)\end{array}$ & & & & \\
\hline \multicolumn{6}{|l|}{ Treatment } \\
\hline Yes & $\begin{array}{l}16.08 \% \\
(303 / 1884)\end{array}$ & \multirow{2}{*}{$\begin{array}{l}1.04 \\
(0.85- \\
1.27)\end{array}$} & \multirow[t]{2}{*}{0.73} & \multirow{2}{*}{\multicolumn{2}{|c|}{ Insignificance }} \\
\hline No & $\begin{array}{l}15.61 \% \\
(180 / 1153)\end{array}$ & & & & \\
\hline \multicolumn{6}{|l|}{ Interventions } \\
\hline \multicolumn{6}{|l|}{ Biological } \\
\hline Yes & $9.40 \%(49 / 521)$ & \multirow{2}{*}{$\begin{array}{l}0.50 \\
(0.37- \\
0.68)\end{array}$} & \multirow{2}{*}{$\begin{array}{l}< \\
0.01\end{array}$} & \multirow{2}{*}{$\begin{array}{l}0.53 \\
(0.39- \\
0.73)\end{array}$} & \multirow{2}{*}{$\begin{array}{l}<.01 \\
0.01\end{array}$} \\
\hline No & $\begin{array}{l}17.25 \% \\
(434 / 2516)\end{array}$ & & & & \\
\hline \multicolumn{6}{|l|}{ Behavioral } \\
\hline Yes & $19.52 \%(49 / 251)$ & \multirow{2}{*}{$\begin{array}{l}1.32 \\
(0.95- \\
1.83)\end{array}$} & \multirow[t]{2}{*}{0.10} & \multirow{2}{*}{\multicolumn{2}{|c|}{ Insignificance }} \\
\hline No & $\begin{array}{l}15.58 \% \\
(434 / 2786)\end{array}$ & & & & \\
\hline \multicolumn{6}{|l|}{ Device } \\
\hline Yes & 18.59\% (37/199) & \multirow{2}{*}{$\begin{array}{l}1.23 \\
(0.85- \\
1.78)\end{array}$} & \multirow[t]{2}{*}{0.28} & \multirow{2}{*}{\multicolumn{2}{|c|}{ Insignificance }} \\
\hline No & $\begin{array}{l}15.72 \% \\
(446 / 2838)\end{array}$ & & & & \\
\hline Diagnostic test & & & & & \\
\hline
\end{tabular}




\begin{tabular}{|c|c|c|c|c|}
\hline \multirow[t]{2}{*}{ Characteristics } & \multirow[t]{2}{*}{$\begin{array}{l}\text { Completed } \\
\text { recruitment rates }\end{array}$} & \multicolumn{2}{|c|}{$\begin{array}{l}\text { Simple logistic } \\
\text { regression }\end{array}$} & $\begin{array}{l}\text { Multiple logistic } \\
\text { regression }\end{array}$ \\
\hline & & $\begin{array}{l}\text { OR }(95 \% \\
\mathrm{Cl})\end{array}$ & $\mathbf{P}$ & $\begin{array}{l}\mathrm{OR}(95 \% \\
\mathrm{Cl})\end{array}$ \\
\hline Yes & $24.59 \%(30 / 122)$ & \multirow{2}{*}{$\begin{array}{l}1.77 \\
(1.16- \\
2.71)\end{array}$} & \multirow{2}{*}{$<.01$} & \multirow[t]{2}{*}{ Insignificance } \\
\hline No & $\begin{array}{l}15.54 \% \\
(453 / 2915)\end{array}$ & & & \\
\hline \multicolumn{5}{|l|}{ Drug } \\
\hline Yes & $\begin{array}{l}15.99 \% \\
(219 / 1370)\end{array}$ & \multirow{2}{*}{$\begin{array}{l}1.01 \\
(0.83- \\
1.23)\end{array}$} & \multirow[t]{2}{*}{0.91} & \multirow[t]{2}{*}{ Insignificance } \\
\hline No & $\begin{array}{l}15.84 \% \\
(264 / 1667)\end{array}$ & & & \\
\hline \multicolumn{5}{|l|}{ Phases } \\
\hline Not applicable & $\begin{array}{l}19.60 \% \\
(224 / 1143)\end{array}$ & \multirow{5}{*}{$\begin{array}{l}0.86 \\
(0.80- \\
0.93)\end{array}$} & \multirow[t]{5}{*}{$<0.01$} & \multirow[t]{5}{*}{ Insignificance } \\
\hline Phase 1 & $15.53 \%(41 / 264)$ & & & \\
\hline Phase 2 & $13.11 \%(111 / 847)$ & & & \\
\hline Phase 3 & $13.02 \%(82 / 630)$ & & & \\
\hline Phase 4 & $16.34 \%(25 / 153)$ & & & \\
\hline
\end{tabular}

\section{Risk Factors for Completion Time}

According to the multivariate analysis for completion time in the entire studies, enrollment $(H R=0.87$, $95 \% \mathrm{Cl}: 0.86-0.89, \mathrm{P}<0.01$, Table 5), funded by NIH (HR $=0.59,95 \% \mathrm{Cl}: 0.50-0.70, \mathrm{P}<0.01)$, and outcome measures including survival $(\mathrm{HR}=0.87,95 \% \mathrm{Cl}: 0.76-1.00, \mathrm{P}=0.05)$ were significant. Namely, smaller enrollment was associated with shorter completion time, while being funded by $\mathrm{NIH}$ and outcome measures including survival were associated with longer completion time. Kaplan-Meier curves for each significant variables based on the univariate analysis are presented in the Fig. 4. 
Table 5

Univariate and multivariate analyses of characteristics for completion time in studies relating to COVID19.

\begin{tabular}{|c|c|c|c|c|c|}
\hline \multirow[t]{2}{*}{ Characteristics } & \multirow[t]{2}{*}{$\begin{array}{l}\text { Time interval (median } \\
(95 \% \mathrm{Cl}) \text {, days) }\end{array}$} & \multicolumn{2}{|l|}{$\begin{array}{l}\text { Simple Cox } \\
\text { regression }\end{array}$} & \multicolumn{2}{|c|}{$\begin{array}{l}\text { Multiple Cox } \\
\text { regression }\end{array}$} \\
\hline & & $\begin{array}{l}\mathrm{HR}(95 \% \\
\mathrm{Cl})\end{array}$ & $\mathbf{P}$ & $\begin{array}{l}\mathrm{HR}(95 \% \\
\mathrm{Cl})\end{array}$ & $\mathbf{P}$ \\
\hline \multicolumn{6}{|l|}{ Gender } \\
\hline Male & $167.00(60.09-273.91)$ & \multirow{3}{*}{$\begin{array}{l}0.91 \\
(0.81- \\
1.030)\end{array}$} & \multirow[t]{3}{*}{0.14} & \multirow{3}{*}{\multicolumn{2}{|c|}{ Insignificance }} \\
\hline Female & $\begin{array}{l}287.00(213.25- \\
360.75)\end{array}$ & & & & \\
\hline Both & $\begin{array}{l}278.00(268.18- \\
287.82)\end{array}$ & & & & \\
\hline \multicolumn{6}{|c|}{ Participants including child } \\
\hline Yes & $\begin{array}{l}275.00(249.79- \\
300.21)\end{array}$ & \multirow{2}{*}{$\begin{array}{l}0.86 \\
(0.81- \\
0.93)\end{array}$} & \multirow[t]{2}{*}{$\dot{0} 0.01$} & \multirow{2}{*}{\multicolumn{2}{|c|}{ Insignificance }} \\
\hline No & $\begin{array}{l}277.00(266.65- \\
287.35)\end{array}$ & & & & \\
\hline \multicolumn{6}{|c|}{$\begin{array}{l}\text { Participants including older } \\
\text { adult }\end{array}$} \\
\hline Yes & $\begin{array}{l}282.00(271.45- \\
292.55)\end{array}$ & \multirow[t]{2}{*}{$\begin{array}{l}0.91(0.83- \\
1.00)\end{array}$} & \multirow[t]{2}{*}{0.05} & \multirow{2}{*}{\multicolumn{2}{|c|}{ Insignificance }} \\
\hline No & $\begin{array}{l}239.00(212.03- \\
265.97)\end{array}$ & & & & \\
\hline \multicolumn{6}{|l|}{ Enrollment } \\
\hline$\leq 50$ & $\begin{array}{l}221.00(206.55- \\
235.45)\end{array}$ & \multirow{5}{*}{$\begin{array}{l}0.87 \\
(0.85- \\
0.89)\end{array}$} & \multirow[t]{5}{*}{ 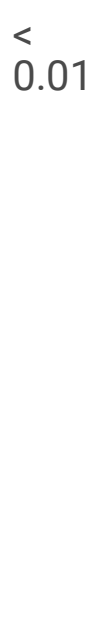 } & \multirow{5}{*}{$\begin{array}{l}0.87 \\
(0.86- \\
0.89)\end{array}$} & \multirow[t]{5}{*}{$\dot{0} 01$} \\
\hline$>50$ and $\leq 100$ & $\begin{array}{l}249.00(233.98- \\
264.02)\end{array}$ & & & & \\
\hline$>100$ and $\leq 300$ & $\begin{array}{l}274.00(257.46- \\
290.54)\end{array}$ & & & & \\
\hline$>300$ and $\leq 900$ & $\begin{array}{l}304.00(278.74- \\
329.26)\end{array}$ & & & & \\
\hline$>900$ & $\begin{array}{l}365.00(350.18- \\
379.82)\end{array}$ & & & & \\
\hline \multicolumn{6}{|c|}{ Funded by industry } \\
\hline Yes & $\begin{array}{l}289.00(272.75- \\
305.25)\end{array}$ & $\begin{array}{l}1.08 \\
(1.01- \\
1.16)\end{array}$ & 0.02 & \multicolumn{2}{|c|}{ Insignificance } \\
\hline
\end{tabular}




\begin{tabular}{|c|c|c|c|c|c|}
\hline \multirow[t]{2}{*}{ Characteristics } & \multirow[t]{2}{*}{$\begin{array}{l}\text { Time interval (median } \\
(95 \% \mathrm{Cl}) \text {, days) }\end{array}$} & \multicolumn{2}{|c|}{$\begin{array}{l}\text { Simple Cox } \\
\text { regression }\end{array}$} & \multicolumn{2}{|c|}{$\begin{array}{l}\text { Multiple Cox } \\
\text { regression }\end{array}$} \\
\hline & & $\begin{array}{l}\mathrm{HR}(95 \% \\
\mathrm{Cl})\end{array}$ & $P$ & $\begin{array}{l}\mathrm{HR}(95 \% \\
\mathrm{Cl})\end{array}$ & $\mathbf{P}$ \\
\hline No & $\begin{array}{l}275.00(264.37- \\
285.63)\end{array}$ & & & & \\
\hline \multicolumn{6}{|l|}{ Funded by NIH } \\
\hline Yes & $\begin{array}{l}471.00(397.49- \\
544.52)\end{array}$ & \multirow{2}{*}{$\begin{array}{l}0.55 \\
(0.47- \\
0.65)\end{array}$} & \multirow[t]{2}{*}{$\dot{0} 01$} & \multirow{2}{*}{$\begin{array}{l}0.59 \\
(0.50- \\
0.70)\end{array}$} & \multirow[t]{2}{*}{$<0.01$} \\
\hline No & $\begin{array}{l}274.00(265.13- \\
282.87)\end{array}$ & & & & \\
\hline \multicolumn{6}{|l|}{ Study type } \\
\hline Interventional & $\begin{array}{l}276.00(264.43- \\
287.57)\end{array}$ & \multirow{2}{*}{$\begin{array}{l}1.14 \\
(1.08- \\
1.21)\end{array}$} & \multirow[t]{2}{*}{$<.01$} & \multirow{2}{*}{\multicolumn{2}{|c|}{ Insignificance }} \\
\hline Observational & $\begin{array}{l}279.00(262.11- \\
295.89)\end{array}$ & & & & \\
\hline \multicolumn{6}{|c|}{$\begin{array}{l}\text { Outcome measures including } \\
\text { ICU stay }\end{array}$} \\
\hline Yes & $\begin{array}{l}255.00(199.73- \\
310.27)\end{array}$ & \multirow{2}{*}{$\begin{array}{l}1.10 \\
(0.93- \\
1.30)\end{array}$} & \multirow[t]{2}{*}{0.27} & \multirow{2}{*}{\multicolumn{2}{|c|}{ Insignificance }} \\
\hline No & $\begin{array}{l}277.00(267.06- \\
286.94)\end{array}$ & & & & \\
\hline \multicolumn{6}{|c|}{$\begin{array}{l}\text { Outcome measures including } \\
\text { length of hospital stay }\end{array}$} \\
\hline Yes & $\begin{array}{l}244.00(217.67- \\
270.33)\end{array}$ & \multirow{2}{*}{$\begin{array}{l}1.09 \\
(0.96- \\
1.24)\end{array}$} & \multirow[t]{2}{*}{0.18} & \multirow{2}{*}{\multicolumn{2}{|c|}{ Insignificance }} \\
\hline No & $\begin{array}{l}280.00(269.86- \\
290.14)\end{array}$ & & & & \\
\hline \multicolumn{6}{|c|}{$\begin{array}{l}\text { Outcome measures including } \\
\text { mortality }\end{array}$} \\
\hline Yes & $\begin{array}{l}294.00(280.07- \\
307.93)\end{array}$ & \multirow{2}{*}{$\begin{array}{l}0.99 \\
(0.94- \\
1.06)\end{array}$} & \multirow[t]{2}{*}{0.82} & \multirow{2}{*}{\multicolumn{2}{|c|}{ Insignificance }} \\
\hline No & $\begin{array}{l}274.00(262.64- \\
285.36)\end{array}$ & & & & \\
\hline \multicolumn{6}{|c|}{$\begin{array}{l}\text { Outcome measures including } \\
\text { survival }\end{array}$} \\
\hline Yes & $\begin{array}{l}346.00(309.99- \\
382.01)\end{array}$ & $\begin{array}{l}0.93 \\
(0.81- \\
1.06)\end{array}$ & 0.28 & $\begin{array}{l}0.87 \\
(0.76- \\
1.00)\end{array}$ & 0.05 \\
\hline
\end{tabular}




\begin{tabular}{|c|c|c|c|c|c|}
\hline \multirow[t]{2}{*}{ Characteristics } & \multirow[t]{2}{*}{$\begin{array}{l}\text { Time interval (median } \\
(95 \% \mathrm{Cl}) \text {, days) }\end{array}$} & \multicolumn{2}{|c|}{$\begin{array}{l}\text { Simple Cox } \\
\text { regression }\end{array}$} & \multicolumn{2}{|c|}{$\begin{array}{l}\text { Multiple Cox } \\
\text { regression }\end{array}$} \\
\hline & & $\begin{array}{l}\mathrm{HR}(95 \% \\
\mathrm{Cl})\end{array}$ & $\mathbf{P}$ & $\begin{array}{l}\mathrm{HR}(95 \% \\
\mathrm{Cl})\end{array}$ & $\mathbf{P}$ \\
\hline No & $\begin{array}{l}275.00(265.86- \\
284.14)\end{array}$ & & & & \\
\hline
\end{tabular}

Based on the multivariate analysis for completion time in the interventional studies, Participants including child ( $\mathrm{HR}=0.88,95 \% \mathrm{Cl}: 0.78-1.00, \mathrm{P}=0.04$, Table 6), enrollment $(\mathrm{HR}=0.87,95 \% \mathrm{Cl}: 0.85-0.90, \mathrm{P}$ $<0.01)$, funded by industry ( $\mathrm{HR}=1.11,95 \% \mathrm{Cl}: 1.02-1.20, \mathrm{P}=0.01)$, funded by $\mathrm{NIH}(\mathrm{HR}=0.69,95 \% \mathrm{Cl}$ : $0.55-0.86, \mathrm{P}<0.01)$, intervention model $(\mathrm{HR}=1.09,95 \% \mathrm{Cl}: 1.05-1.14, \mathrm{P}<0.01)$, basic science $(\mathrm{HR}=0.57$, $95 \% \mathrm{Cl}: 0.40-0.79, \mathrm{P}<0.01)$, device feasibility $(\mathrm{HR}=3.41,95 \% \mathrm{Cl}: 1.70-6.86, \mathrm{P}<0.01)$, treatment $(\mathrm{HR}=$ $0.92,95 \% \mathrm{Cl}: 0.85-0.99, \mathrm{P}=0.03)$, and biological interventions ( $\mathrm{HR}=0.75,95 \% \mathrm{Cl}: 0.68-0.83, \mathrm{P}<0.01)$ were found to have significance. This was to say that Participants including child, smaller enrollment, crossover intervention model, and primary purpose involving in device feasibility and treatment were associated with shorter completion time, while being funded by industry and $\mathrm{NIH}$, primary purpose involving in basic science, and biological interventions were associated with longer completion time. Kaplan-Meier curves for each significant variables based on the univariate analysis are presented in the Fig. 5. 
Table 6

Univariate and multivariate analyses of characteristics for completed time in interventional studies relating to COVID-19.

\begin{tabular}{|c|c|c|c|c|c|}
\hline \multirow[t]{2}{*}{ Characteristics } & \multirow[t]{2}{*}{$\begin{array}{l}\text { Median, 95\%Cl } \\
\text { (days) }\end{array}$} & \multicolumn{2}{|c|}{$\begin{array}{l}\text { Simple Cox } \\
\text { regression }\end{array}$} & \multicolumn{2}{|l|}{$\begin{array}{l}\text { Multiple Cox } \\
\text { regression }\end{array}$} \\
\hline & & $\begin{array}{l}\mathrm{HR}(95 \% \\
\mathrm{Cl})\end{array}$ & $\mathbf{P}$ & $\begin{array}{l}\mathrm{HR}(95 \% \\
\mathrm{Cl})\end{array}$ & $\mathbf{P}$ \\
\hline \multicolumn{6}{|l|}{ Gender } \\
\hline Male & $\begin{array}{l}120.00(88.35- \\
151.65)\end{array}$ & \multirow{3}{*}{$\begin{array}{l}0.89 \\
(0.75- \\
1.05)\end{array}$} & \multirow[t]{3}{*}{0.16} & \multirow{3}{*}{\multicolumn{2}{|c|}{ Insignificance }} \\
\hline Female & $\begin{array}{l}287.00(116.34- \\
457.66)\end{array}$ & & & & \\
\hline Both & $\begin{array}{l}276.00(264.39- \\
287.61)\end{array}$ & & & & \\
\hline \multicolumn{6}{|c|}{ Participants including child } \\
\hline Yes & $\begin{array}{l}275.00(229.07- \\
320.93)\end{array}$ & \multirow{2}{*}{$\begin{array}{l}0.82 \\
(0.72- \\
0.92)\end{array}$} & \multirow[t]{2}{*}{ <. 01} & \multirow[t]{2}{*}{$\begin{array}{l}0.88(0.78- \\
1.00)\end{array}$} & \multirow[t]{2}{*}{0.04} \\
\hline No & $\begin{array}{l}276.00(264.18- \\
287.82)\end{array}$ & & & & \\
\hline \multicolumn{6}{|c|}{ Participants including older adult } \\
\hline Yes & $\begin{array}{l}278.00(265.88- \\
290.13)\end{array}$ & \multirow{2}{*}{$\begin{array}{l}0.89 \\
(0.78- \\
1.01)\end{array}$} & \multirow[t]{2}{*}{0.07} & \multirow[t]{2}{*}{ Insignificance } & \\
\hline No & $\begin{array}{l}254.00(221.14- \\
286.86)\end{array}$ & & & & \\
\hline \multicolumn{6}{|l|}{ Enrollment } \\
\hline$\leq 50$ & $\begin{array}{l}230.00(214.65- \\
245.35)\end{array}$ & \multirow{5}{*}{$\begin{array}{l}0.88 \\
(0.86- \\
0.90)\end{array}$} & \multirow[t]{5}{*}{$\begin{array}{l}< \\
0.01\end{array}$} & \multirow{5}{*}{$\begin{array}{l}0.87 \\
(0.85- \\
0.90)\end{array}$} & \multirow[t]{5}{*}{$\begin{array}{l}< \\
0.01\end{array}$} \\
\hline$>50$ and $\leq 100$ & $\begin{array}{l}252.00(237.38- \\
266.62)\end{array}$ & & & & \\
\hline$>100$ and $\leq 300$ & $\begin{array}{l}280.00(258.15- \\
301.85)\end{array}$ & & & & \\
\hline$>300$ and $\leq 900$ & $\begin{array}{l}327.00(293.65- \\
360.35)\end{array}$ & & & & \\
\hline$>900$ & $\begin{array}{l}375.00 \text { (352.70- } \\
397.30)\end{array}$ & & & & \\
\hline \multicolumn{6}{|c|}{ Funded by industry } \\
\hline Yes & $\begin{array}{l}289.00(272.76- \\
305.24)\end{array}$ & $\begin{array}{l}1.06 \\
(0.98- \\
1.15)\end{array}$ & 0.16 & $\begin{array}{l}1.11 \\
(1.02- \\
1.20)\end{array}$ & 0.01 \\
\hline
\end{tabular}




\begin{tabular}{|c|c|c|c|c|c|}
\hline \multirow[t]{2}{*}{ Characteristics } & \multirow[t]{2}{*}{$\begin{array}{l}\text { Median, 95\%Cl } \\
\text { (days) }\end{array}$} & \multicolumn{2}{|c|}{$\begin{array}{l}\text { Simple Cox } \\
\text { regression }\end{array}$} & \multicolumn{2}{|l|}{$\begin{array}{l}\text { Multiple Cox } \\
\text { regression }\end{array}$} \\
\hline & & $\begin{array}{l}\mathrm{HR}(95 \% \\
\mathrm{Cl})\end{array}$ & $\mathbf{P}$ & $\begin{array}{l}\mathrm{HR}(95 \% \\
\mathrm{Cl})\end{array}$ & $\mathbf{P}$ \\
\hline No & $\begin{array}{l}274.00(260.32- \\
287.68)\end{array}$ & & & & \\
\hline \multicolumn{6}{|l|}{ Funded by $\mathrm{NIH}$} \\
\hline Yes & $\begin{array}{l}423.00(335.71- \\
510.29)\end{array}$ & \multirow{2}{*}{$\begin{array}{l}0.64 \\
(0.52- \\
0.80)\end{array}$} & \multirow[t]{2}{*}{$\dot{0} 01$} & \multirow{2}{*}{$\begin{array}{l}0.69 \\
(0.55- \\
0.86)\end{array}$} & \multirow[t]{2}{*}{$<.01$} \\
\hline No & $\begin{array}{l}275.00(263.60- \\
286.40)\end{array}$ & & & & \\
\hline \multicolumn{6}{|c|}{$\begin{array}{l}\text { Outcome measures including ICU } \\
\text { stay }\end{array}$} \\
\hline Yes & $\begin{array}{l}247.00(196.64- \\
297.36)\end{array}$ & \multirow{2}{*}{$\begin{array}{l}1.13 \\
(0.92- \\
1.39)\end{array}$} & \multirow[t]{2}{*}{0.25} & \multirow{2}{*}{\multicolumn{2}{|c|}{ Insignificance }} \\
\hline No & $\begin{array}{l}277.00(265.30- \\
288.70)\end{array}$ & & & & \\
\hline \multicolumn{6}{|c|}{$\begin{array}{l}\text { Outcome measures including length } \\
\text { of hospital stay }\end{array}$} \\
\hline Yes & $\begin{array}{l}244.00(209.76- \\
278.24)\end{array}$ & \multirow{2}{*}{$\begin{array}{l}1.07 \\
(0.92- \\
1.24)\end{array}$} & \multirow[t]{2}{*}{0.41} & \multirow{2}{*}{\multicolumn{2}{|c|}{ Insignificance }} \\
\hline No & $\begin{array}{l}280.00 \text { (268.03- } \\
291.97)\end{array}$ & & & & \\
\hline \multicolumn{6}{|c|}{$\begin{array}{l}\text { Outcome measures including } \\
\text { mortality }\end{array}$} \\
\hline Yes & $\begin{array}{l}287.00(270.16- \\
303.84)\end{array}$ & \multirow{2}{*}{$\begin{array}{l}0.96 \\
(0.89- \\
1.03)\end{array}$} & \multirow[t]{2}{*}{0.26} & \multirow{2}{*}{\multicolumn{2}{|c|}{ Insignificance }} \\
\hline No & $\begin{array}{l}274.00(260.66- \\
287.34)\end{array}$ & & & & \\
\hline \multicolumn{6}{|c|}{$\begin{array}{l}\text { Outcome measures including } \\
\text { survival }\end{array}$} \\
\hline Yes & $\begin{array}{l}308.00(58.84- \\
357.16)\end{array}$ & \multirow{2}{*}{$\begin{array}{l}0.94 \\
(0.80- \\
1.11)\end{array}$} & \multirow[t]{2}{*}{0.49} & \multirow{2}{*}{\multicolumn{2}{|c|}{ Insignificance }} \\
\hline No & $\begin{array}{l}275.00(263.46- \\
286.54)\end{array}$ & & & & \\
\hline \multicolumn{6}{|l|}{ Allocation } \\
\hline $\mathrm{N} / \mathrm{A}$ & $\begin{array}{l}304.00(266.61- \\
341.39)\end{array}$ & $\begin{array}{l}1.03 \\
(0.99- \\
1.08)\end{array}$ & 0.15 & Insignificanc & \\
\hline
\end{tabular}









\begin{tabular}{|c|c|c|c|c|c|}
\hline \multirow[t]{2}{*}{ Characteristics } & \multirow[t]{2}{*}{$\begin{array}{l}\text { Median, 95\%Cl } \\
\text { (days) }\end{array}$} & \multicolumn{2}{|c|}{$\begin{array}{l}\text { Simple Cox } \\
\text { regression }\end{array}$} & \multicolumn{2}{|l|}{$\begin{array}{l}\text { Multiple Cox } \\
\text { regression }\end{array}$} \\
\hline & & $\begin{array}{l}\mathrm{HR}(95 \% \\
\mathrm{Cl})\end{array}$ & $\mathbf{P}$ & $\begin{array}{l}\mathrm{HR}(95 \% \\
\mathrm{Cl})\end{array}$ & $\mathbf{P}$ \\
\hline Yes & $\begin{array}{l}59.00(0.00- \\
135.23)\end{array}$ & \multirow{2}{*}{$\begin{array}{l}4.28 \\
(2.14- \\
8.57)\end{array}$} & \multirow[t]{2}{*}{ <. 01} & \multirow{2}{*}{$\begin{array}{l}3.41 \\
(1.70- \\
6.86)\end{array}$} & \multirow[t]{2}{*}{$\begin{array}{l}< \\
0.01\end{array}$} \\
\hline No & $\begin{array}{l}276.00(264.51- \\
287.49)\end{array}$ & & & & \\
\hline \multicolumn{6}{|l|}{ Diagnostic test } \\
\hline Yes & $\begin{array}{l}304.00(231.75- \\
376.25)\end{array}$ & \multirow{2}{*}{$\begin{array}{l}0.97 \\
(0.82- \\
1.15)\end{array}$} & \multirow[t]{2}{*}{0.73} & \multirow{2}{*}{\multicolumn{2}{|c|}{ Insignificance }} \\
\hline No & $\begin{array}{l}276.00(264.42- \\
287.59)\end{array}$ & & & & \\
\hline \multicolumn{6}{|c|}{ Health services research } \\
\hline Yes & $\begin{array}{l}289.00(248.33- \\
329.67)\end{array}$ & \multirow{2}{*}{$\begin{array}{l}0.88 \\
(0.71- \\
1.09)\end{array}$} & \multirow[t]{2}{*}{0.24} & \multirow{2}{*}{\multicolumn{2}{|c|}{ Insignificance }} \\
\hline No & $\begin{array}{l}276.00(264.11- \\
287.90)\end{array}$ & & & & \\
\hline \multicolumn{6}{|l|}{ Prevention } \\
\hline Yes & $\begin{array}{l}349.00(324.83- \\
373.17)\end{array}$ & \multirow{2}{*}{$\begin{array}{l}0.86 \\
(0.78- \\
0.94)\end{array}$} & \multirow[t]{2}{*}{$\begin{array}{l}<.01 \\
0.01\end{array}$} & \multirow{2}{*}{\multicolumn{2}{|c|}{ Insignificance }} \\
\hline No & $\begin{array}{l}271.00(259.41- \\
282.59)\end{array}$ & & & & \\
\hline \multicolumn{6}{|l|}{ Screening } \\
\hline Yes & $\begin{array}{l}284.00(90.93- \\
477.07)\end{array}$ & \multirow{2}{*}{$\begin{array}{l}0.84 \\
(0.61- \\
1.17)\end{array}$} & \multirow[t]{2}{*}{0.30} & \multirow{2}{*}{\multicolumn{2}{|c|}{ Insignificance }} \\
\hline No & $\begin{array}{l}276.00(264.49- \\
287.52)\end{array}$ & & & & \\
\hline \multicolumn{6}{|l|}{ Supportive care } \\
\hline Yes & $\begin{array}{l}243.00(197.24- \\
288.76)\end{array}$ & \multirow{2}{*}{$\begin{array}{l}1.21 \\
(1.03- \\
1.43)\end{array}$} & \multirow[t]{2}{*}{0.02} & \multirow{2}{*}{\multicolumn{2}{|c|}{ Insignificance }} \\
\hline No & $\begin{array}{l}278.00(266.50- \\
289.50)\end{array}$ & & & & \\
\hline \multicolumn{6}{|l|}{ Treatment } \\
\hline Yes & $\begin{array}{l}269.00(256.59- \\
281.41)\end{array}$ & $\begin{array}{l}1.09 \\
(1.01- \\
1.17)\end{array}$ & 0.02 & $\begin{array}{l}0.92 \\
(0.85- \\
0.99)\end{array}$ & 0.03 \\
\hline
\end{tabular}




\begin{tabular}{|c|c|c|c|c|c|}
\hline \multirow[t]{2}{*}{ Characteristics } & \multirow[t]{2}{*}{$\begin{array}{l}\text { Median, 95\%Cl } \\
\text { (days) }\end{array}$} & \multicolumn{2}{|l|}{$\begin{array}{l}\text { Simple Cox } \\
\text { regression }\end{array}$} & \multicolumn{2}{|l|}{$\begin{array}{l}\text { Multiple Cox } \\
\text { regression }\end{array}$} \\
\hline & & $\begin{array}{l}\mathrm{HR}(95 \% \\
\mathrm{Cl})\end{array}$ & $\mathbf{P}$ & $\begin{array}{l}\mathrm{HR}(95 \% \\
\mathrm{Cl})\end{array}$ & $\mathbf{P}$ \\
\hline No & $\begin{array}{l}305.00(284.37- \\
325.63)\end{array}$ & & & & \\
\hline \multicolumn{6}{|l|}{ Interventions } \\
\hline \multicolumn{6}{|l|}{ Biological } \\
\hline Yes & $\begin{array}{l}365.00(344.56- \\
385.44)\end{array}$ & \multirow{2}{*}{$\begin{array}{l}0.75 \\
(0.68- \\
0.82)\end{array}$} & \multirow[t]{2}{*}{$\begin{array}{l}<.01 \\
0.01\end{array}$} & \multirow{2}{*}{$\begin{array}{l}0.75 \\
(0.68- \\
0.83)\end{array}$} & \multirow[t]{2}{*}{$\dot{0.01}$} \\
\hline No & $\begin{array}{l}260.00(249.31- \\
270.69)\end{array}$ & & & & \\
\hline \multicolumn{6}{|l|}{ Behavioral } \\
\hline Yes & $\begin{array}{l}262.00(226.35- \\
297.65)\end{array}$ & \multirow{2}{*}{$\begin{array}{l}0.99 \\
(0.87- \\
1.13)\end{array}$} & \multirow[t]{2}{*}{0.92} & \multirow{2}{*}{\multicolumn{2}{|c|}{ Insignificance }} \\
\hline No & $\begin{array}{l}277.00(265.06- \\
288.94)\end{array}$ & & & & \\
\hline \multicolumn{6}{|l|}{ Device } \\
\hline Yes & $\begin{array}{l}245.00(189.11- \\
300.89)\end{array}$ & \multirow{2}{*}{$\begin{array}{l}1.25 \\
(1.08- \\
1.45)\end{array}$} & \multirow[t]{2}{*}{ <. 01} & \multirow{2}{*}{\multicolumn{2}{|c|}{ Insignificance }} \\
\hline No & $\begin{array}{l}277.00(265.32- \\
288.68)\end{array}$ & & & & \\
\hline \multicolumn{6}{|l|}{ Diagnostic test } \\
\hline Yes & $\begin{array}{l}269.00 \text { (168.49- } \\
369.51)\end{array}$ & \multirow{2}{*}{$\begin{array}{l}0.97 \\
(0.81- \\
1.16)\end{array}$} & \multirow[t]{2}{*}{0.74} & \multirow{2}{*}{\multicolumn{2}{|c|}{ Insignificance }} \\
\hline No & $\begin{array}{l}276.00(264.34- \\
287.66)\end{array}$ & & & & \\
\hline \multicolumn{6}{|l|}{ Drug } \\
\hline Yes & $\begin{array}{l}258.00 \\
269.75)\end{array}$ & \multirow{2}{*}{$\begin{array}{l}1.16 \\
(1.08- \\
1.24)\end{array}$} & \multirow[t]{2}{*}{ <. 01} & \multirow{2}{*}{\multicolumn{2}{|c|}{ Insignificance }} \\
\hline No & $\begin{array}{l}304.00 \text { (288.77- } \\
319.23)\end{array}$ & & & & \\
\hline \multicolumn{6}{|l|}{ Phases } \\
\hline Not applicable & $\begin{array}{l}254.00(233.78- \\
274.22)\end{array}$ & \multirow[t]{2}{*}{$\begin{array}{l}0.97(0.95- \\
1.00)\end{array}$} & \multirow[t]{2}{*}{0.04} & \multirow{2}{*}{\multicolumn{2}{|c|}{ Insignificance }} \\
\hline Phase 1 & $\begin{array}{l}274.00(238.17- \\
309.83)\end{array}$ & & & & \\
\hline
\end{tabular}




\begin{tabular}{|c|c|c|c|c|c|}
\hline \multirow[t]{2}{*}{ Characteristics } & \multirow[t]{2}{*}{$\begin{array}{l}\text { Median, 95\% Cl } \\
\text { (days) }\end{array}$} & \multicolumn{2}{|c|}{$\begin{array}{l}\text { Simple Cox } \\
\text { regression }\end{array}$} & \multicolumn{2}{|c|}{$\begin{array}{l}\text { Multiple Cox } \\
\text { regression }\end{array}$} \\
\hline & & $\begin{array}{l}\mathrm{HR}(95 \% \\
\mathrm{Cl})\end{array}$ & $\mathbf{P}$ & $\begin{array}{l}\mathrm{HR}(95 \% \\
\mathrm{Cl})\end{array}$ & $\mathbf{P}$ \\
\hline Phase 2 & $\begin{array}{l}305.00(285.00- \\
325.00)\end{array}$ & & & & \\
\hline Phase 3 & $\begin{array}{l}276.00(255.81- \\
296.19)\end{array}$ & & & & \\
\hline Phase 4 & $\begin{array}{l}281.00(228.24- \\
333.76)\end{array}$ & & & & \\
\hline
\end{tabular}

\section{Association between Recruitment Status and Completion time}

In the entire sample, studies with a completed status (median time $=97.00,95 \% \mathrm{Cl}$ : 90.66-103.34 days) had a significant shorter completion time as compared with studies with other status (median time = 349.00, 95\% Cl: 342.89-355.11 days) (Fig. 6A, P<0.01, log-rank test). Regarding the analysis for all interventional studies, similar trend was also observed among studies with a completed status (median time $=122.00,95 \% \mathrm{Cl}: 112.06-131.94$ days) vs. other status (median time $=333.00,95 \% \mathrm{Cl}: 322.08-343.92$ days) (Fig. 6B, $\mathrm{P}<0.01$, log-rank test).

\section{Discussion}

This study was the first to describe the whole trends of the number of COVID-19-related clinical studies registered at ClinicalTrial.gov and we find that the number of studies increased exponentially from January to April in 2020 after the pandemic breakout and reached its peak at April 2020 with about more than 800 clinical studies registered at ClinicalTrial.gov that month. After August 2020, the number of clinical studies remained stable, ranging from 200 to 300. Regarding the number of COVID-19 patients, it rapidly increased up to 20 million cases in November 2020 from about 2000 cases in January 2020. After reaching its first peak in November 2020, the number gradually has decreased until February 2021 and then went up again. The peak of the number of clinical studies relating to COVID-19 was seven months earlier than the first peak of the number of COVID-19 cases, which indicated that the response to the great pandemic was rapid and timely and it may save thousands of lives.

The rate of completed recruitment in our study was overall $19.59 \%$, and $15.9 \%$ in exclusively interventional studies, and this is consistent with the rates in other clinical studies using the ClinicalTrials.gov database $[16,17]$. Currently, it is rather clear that the majority of researches that could not be completed normally because of poor recruitment or other reasons, including preliminary safety and efficacy findings or changes in the standard of care that occurred after the study had been initiated $[5,18$, 
19]. Such termination likely wastes medical resources and may be unavoidable to some extent. However, there may be opportunities to reduce medical waste, such as improving study design and coordination issues with trial conduct $[20,21]$.

Our article focused on evaluating the factors impacting on the recruitment rates and completion time of clinical researches registered in ClinicalTrials.gov and showed that participants only including male, Participants including child, smaller enrollment, not being funded by industry and/or $\mathrm{NIH}$, and observational studies were tend to be relevant to higher completed recruitment rates. In addition, smaller enrollment, none masking, primary purpose having diagnostic test, and non-biological interventions were identified as independent contributors of higher completed recruitment rates in interventional studies.

There is a usual belief that the younger the age, the more difficult it would to be recruited in a research project. Nevertheless, our results demonstrated that the probability of completing a trial was higher in clinical researches recruiting children, compared to those recruiting exclusively adults, which was consistent with the study conducted by Kasenda et al. [17]. That is to say, although challenges with regard to research participation and conduct of pediatric clinical researches differ from those encountered in adults, they do not necessarily compromise the completion of a trial when appropriately addressed $[22,23]$. Indirectly, our results suggested that participants' young age was not related to such recruitment difficulties. It is also possible that because of the specific challenges and the vulnerability of children especially with COVID-19, pediatric clinical researches are more carefully planned and conducted [23].

Generally speaking, industry funding is thought to favor completion rates in a clinical research [17], and the adequacy of financial resources is more likely to ensure feasibility before trial initiation. However, in our article, both $\mathrm{NIH}$ and industry sponsors contributing to lower rate of completion and inefficiencies of clinical researches. Given the seriousness and particularity of COVID-19, countries attach great importance to prevention and control of the epidemic and are eager to find effective treatment or prevention methods at an early date. Therefore, they have strengthened financial support for relevant clinical researches. People usually have higher expectations on these clinical researches, they are usually designed in a more thorough and reasonable way at the beginning of the design, they rely on national medical treatment institutions, have sufficient recruitment sources and larger recruitment project. Due to the large workload and more detailed implementation process, the time to complete recruitment is correspondingly extended. This is consistent with larger enrollment number, longer recruitment times, and faster the recruitment, greater the probability of the final completion of clinical studies.

As for the suggestions for a specific study, the smaller the sample size and the simpler the evaluation process, such as primary purpose involving in diagnostic test, observational studies, non-masking, and non-biological interventions, the higher the possibility of completion of recruitment. This suggests that on the premise of ensuring the effectiveness of experimental evidence, the sample size should be appropriately reduced, if applicable, and the process and evaluation indicators should be simplified, although it is difficult to give consideration to both. Interestingly, observational studies have better rates 
of completion as they are more resource-efficient than interventional trials especially with regard to sample size requirements and probably more positively viewed by participants as they allow access to the necessary and appropriate treatment whether or not under evaluation [24]. Crossover intervention model, and primary purpose involving in device feasibility and treatment were also associated with shorter completion time, while being funded by industry and $\mathrm{NIH}$, primary purpose involving in basic science, and biological interventions were associated with longer completion time. The information may provide significant guides to clinical researchers about how to shorten studies' completion time during the COVID-19 pandemic.

However, our conclusions rely on the quality of the data registered in the ClinicalTrials.gov database and the database does not consist of all clinical trials around the world [25]. And the statistically significant differences are probably due to the use of other trial registries or that of a selection of trials registered in ClinicalTrials.gov, thus it is easy to misuse the information. However, our study included a large number of clinical studies and study design was carefully conducted, thus the results would be relative reliable. Although it is regularly updated by sponsors and investigators, actual research information may not be accurately reflected [26-28]. Another limitation of our study was that elements of trial methodology potentially associated with trial undone due to poor recruitment, eg, recruitment methods, financial or nonfinancial incentives, and study piloting, were frequently not reported and thus limited our risk factor analysis.

\section{Conclusions}

A multitude of clinical studies relating to COVID-19 are registered in responding to the pandemic and the response is rapid and timely, but these clinical studies are frequently not completed. Increased focus on establishing global initiatives and networks to coordinate recruitment efforts may be needed. Several independent risk factors are identified to guide the design of COVID-19-related clinical studies. This may be significant to avoid waste and ensure that the participation of all participants in clinical researches contributes to the treatment or prevention of COVID-19.

\section{Abbreviations}

Cl, confident interval; COVID-19, corona virus disease 2019; HR, hazard rate; ICU, intensive care unit; NIH, national institutes of health; $\mathrm{OR}$, odds rate.

\section{Declarations}

Acknowledgements

None.

\section{Author Contributions}


Conceptualization: Mingxing Lei, Feng Lin.

Data curation: Mingxing Lei, Jinglan Li, Feng Lin.

Formal analysis: Mingxing Lei, Houchen Lv.

Investigation: Mingxing Lei, Houchen Lv.

Methodology: Licheng Zhang, Feng Lin.

Software: Mingxing Lei, Jinglan Li.

Visualization: Mingxing Lei.

Writing - original draft: Mingxing Lei, Jinglan Li.

Writing - review \& editing: All authors.

\section{Funding}

None.

\section{Availability of data and materials}

The datasets used and/or analysed during the current study are available from the corresponding author on reasonable request.

Ethics approval and consent to participate

Not applicable.

\section{Consent for publication}

Not applicable.

\section{Competing interests}

The authors declare that they have no competing interests.

Source(s) of financial support

None.

Conflict of Interest Statements

None. 


\section{References}

1. World Health Organization - WHO. WHO Coronavirus (COVID-19) Dashboard. 2021. Available from: https://covid19.who.int/. Accessed July 6, 2021.

2. Jones CW, Woodford AL, Platts-Mills TF. Characteristics of COVID-19 clinical trials registered with ClinicalTrials.gov: cross-sectional analysis. BMJ open. 2020;10(9):e041276. doi: 10.1136/bmjopen2020-041276. PubMed Central PMCID: PMC32948577.

3. Kasenda B, von Elm E, You J, Bl“" ${ }^{\prime} \mathrm{mle} A$, Tomonaga Y, Saccilotto R, et al. Prevalence, characteristics, and publication of discontinued randomized trials. JAMA. 2014;311(10):1045-51.

4. Dong J, Geng Y, Lu D, Li B, Tian L, Lin D, et al. Clinical Trials for Artificial Intelligence in Cancer Diagnosis: A Cross-Sectional Study of Registered Trials in ClinicalTrials.gov. Frontiers in oncology. 2020;10:1629.

5. Bl" ${ }^{\prime}$ mle A, Schandelmaier S, Oeller P, Kasenda B, Briel M, von Elm E. Premature Discontinuation of Prospective Clinical Studies Approved by a Research Ethics Committee - A Comparison of Randomised and Non-Randomised Studies. PloS one. 2016;11(10):e0165605.

6. Alturki R, Schandelmaier S, Olu KK, von Niederh?usern B, Agarwal A, Frei R, et al. Premature trial discontinuation often not accurately reflected in registries: comparison of registry records with publications. Journal of clinical epidemiology. 2017;81:56-63.

7. Prayle AP, Hurley MN, Smyth AR. Compliance with mandatory reporting of clinical trial results on ClinicalTrials.gov: cross sectional study. BMJ. 2012;344:d7373. Epub 2012/01/05. doi: 10.1136/bmj.d7373. PubMed PMID: 22214756.

8. Ross JS, Tse T, Zarin DA, Xu H, Zhou L, Krumholz HM. Publication of NIH funded trials registered in ClinicalTrials.gov: cross sectional analysis. BMJ. 2012;344:d7292. Epub 2012/01/05. doi: 10.1136/bmj.d7292. PubMed PMID: 22214755; PubMed Central PMCID: PMCPMC3623605.

9. Becker JE, Krumholz HM, Ben-Josef G, Ross JS. Reporting of results in ClinicalTrials.gov and highimpact journals. JAMA. 2014;311(10):1063-5. Epub 2014/03/13. doi: 10.1001/jama.2013.285634. PubMed PMID: 24618969; PubMed Central PMCID: PMCPMC3979514.

10. Glasziou P, Altman DG, Bossuyt P, Boutron I, Clarke M, Julious S, et al. Reducing waste from incomplete or unusable reports of biomedical research. Lancet (London, England). 2014;383(9913):267-76.

11. Moher D, Glasziou P, Chalmers I, Nasser M, Bossuyt PMM, Korevaar DA, et al. Increasing value and reducing waste in biomedical research: who's listening? Lancet (London, England). 2016;387(10027):1573-86.

12. Ehrhardt S, Appel LJ, Meinert CL. Trends in National Institutes of Health Funding for Clinical Trials Registered in ClinicalTrials.gov. JAMA. 2015;314(23):2566-7. Epub 2015/12/17. doi: 10.1001/jama.2015.12206. PubMed PMID: 26670975; PubMed Central PMCID: PMCPMC4919115.

13. Ross JS, Mulvey GK, Hines EM, Nissen SE, Krumholz HM. Trial publication after registration in ClinicalTrials.Gov: a cross-sectional analysis. PLoS medicine. 2009;6(9):e1000144. 
14. Bourgeois FT, Murthy S, Mandl KD. Outcome reporting among drug trials registered in ClinicalTrials.gov. Annals of internal medicine. 2010;153(3):158-66.

15. Jones CW, Safferman MR, Adams AC, Platts-Mills TF. Discrepancies between ClinicalTrials.gov recruitment status and actual trial status: a cross-sectional analysis. BMJ open. 2017;7(10):e017719.

16. Williams RJ, Tse T, DiPiazza K, Zarin DA. Terminated Trials in the ClinicalTrials.gov Results Database: Evaluation of Availability of Primary Outcome Data and Reasons for Termination. PloS one. 2015;10(5):e0127242.

17. Dufetelle $E, t$ Jong GW, Kaguelidou F. Randomized controlled trials in pediatric patients had higher completion rates than adult trials: a cross-sectional study. Journal of clinical epidemiology. 2018;100:53-60.

18. Briel M, Olu KK, von Elm E, Kasenda B, Alturki R, Agarwal A, et al. A systematic review of discontinued trials suggested that most reasons for recruitment failure were preventable. Journal of clinical epidemiology. 2016;80:8-15.

19. Bernardez-Pereira S, Lopes RD, Carrion MJ, Santucci EV, Soares RM, de Oliveira Abreu M, et al. Prevalence, characteristics, and predictors of early termination of cardiovascular clinical trials due to low recruitment: insights from the ClinicalTrials.gov registry. American heart journal. 2014;168(2):213-9.e1.

20. Kasenda B, von Elm EB, You J, B|" ${ }^{* 1}$ mle A, Tomonaga $Y$, Saccilotto R, et al. Learning from failurerationale and design for a study about discontinuation of randomized trials (DISCO study). BMC medical research methodology. 2012;12:131.

21. Campbell MK, Snowdon C, Francis D, Elbourne D, McDonald AM, Knight R, et al. Recruitment to randomised trials: strategies for trial enrollment and participation study. The STEPS study. Health technology assessment (Winchester, England). 2007;11(48):iii, ix-105.

22. van der Lee JH, Wesseling J, Tanck MW, Offringa M. Efficient ways exist to obtain the optimal sample size in clinical trials in rare diseases. Journal of clinical epidemiology. 2008;61(4):324-30.

23. Pica N, Bourgeois F. Discontinuation and Nonpublication of Randomized Clinical Trials Conducted in Children. Pediatrics. 2016;138(3).

24. Califf RM, Zarin DA, Kramer JM, Sherman RE, Aberle LH, Tasneem A. Characteristics of clinical trials registered in ClinicalTrials.gov, 2007-2010. JAMA. 2012;307(17):1838-47. Epub 2012/05/03. doi: 10.1001/jama.2012.3424. PubMed PMID: 22550198.

25. Tse T, Fain KM, Zarin DA. How to avoid common problems when using ClinicalTrials.gov in research: 10 issues to consider. BMJ. 2018;361:k1452. Epub 2018/05/29. doi: 10.1136/bmj.k1452. PubMed PMID: 29802130; PubMed Central PMCID: PMCPMC5968400 declaration of interests and declare the following interests: none.

26. Rees CA, Pica N, Monuteaux MC, Bourgeois FT. Noncompletion and nonpublication of trials studying rare diseases: A cross-sectional analysis. PLoS medicine. 2019;16(11):e1002966.

27. Chapman SJ, Shelton B, Mahmood H, Fitzgerald JE, Harrison EM, Bhangu A. Discontinuation and non-publication of surgical randomised controlled trials: observational study. BMJ (Clinical research 
ed). 2014;349:g6870.

28. Jamjoom AAB, Gane AB, Demetriades AK. Randomized controlled trials in neurosurgery: an observational analysis of trial discontinuation and publication outcome. Journal of neurosurgery. 2017;127(4):857-66.

\section{Figures}

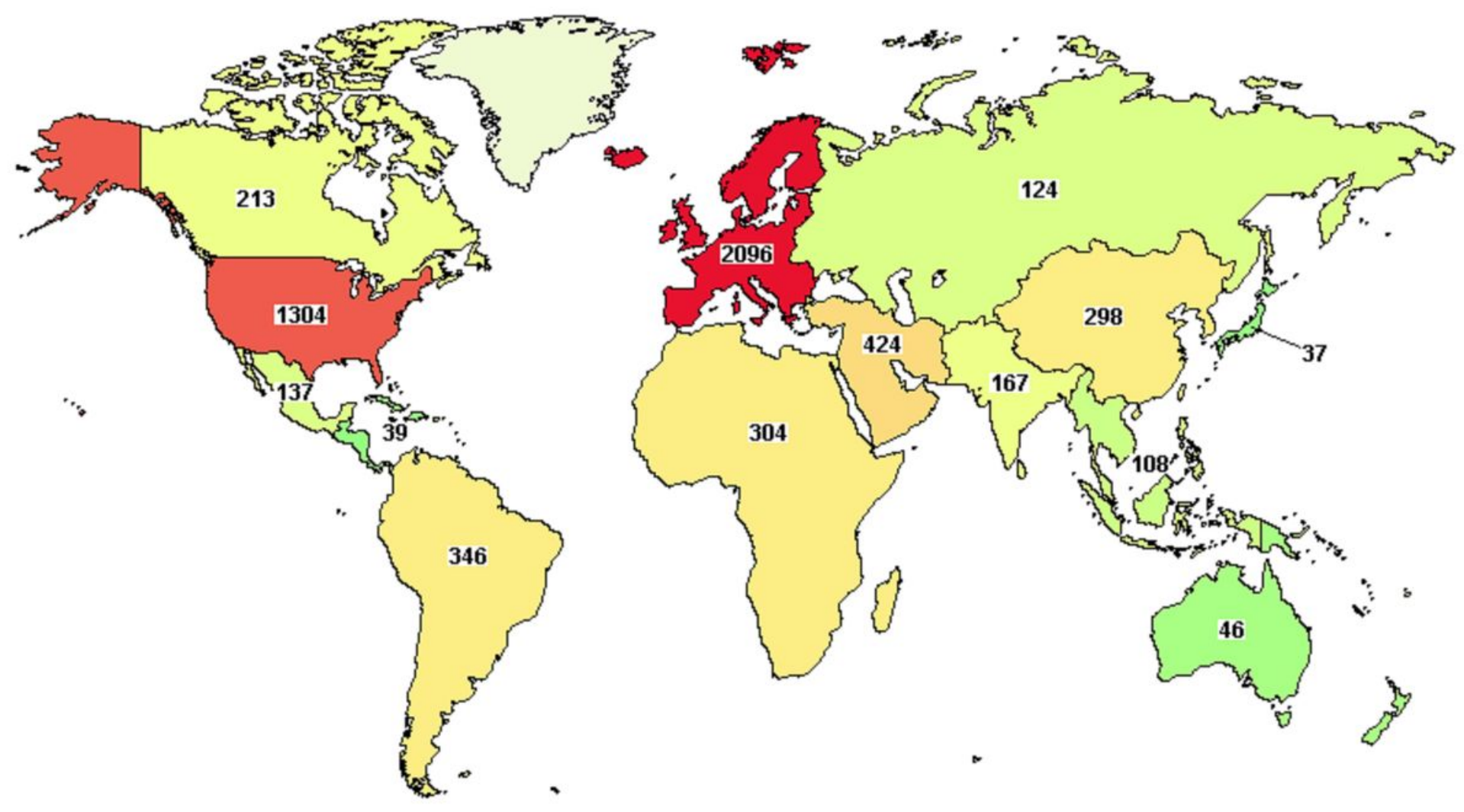

Colors indicate the number of studies with locations in that region.

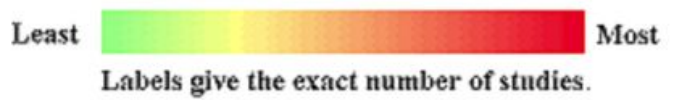

\section{Figure 1}

A map about the number of clinical studies relating to COVID-19 in the whole world (Green indicates the least; Red indicates the most). 


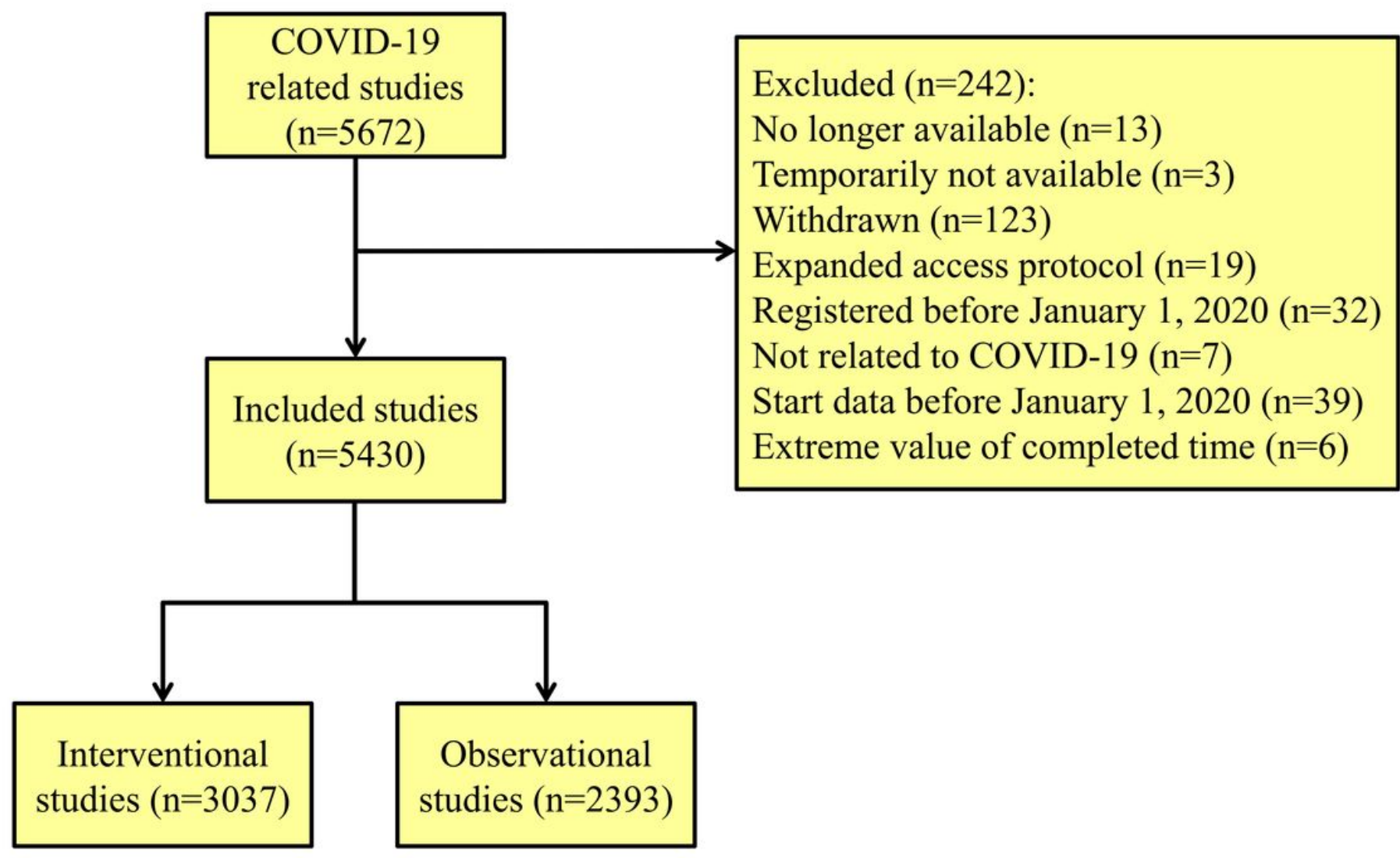

Figure 2

Flow chart of included clinical studies registered at ClinicalTrials.gov. 


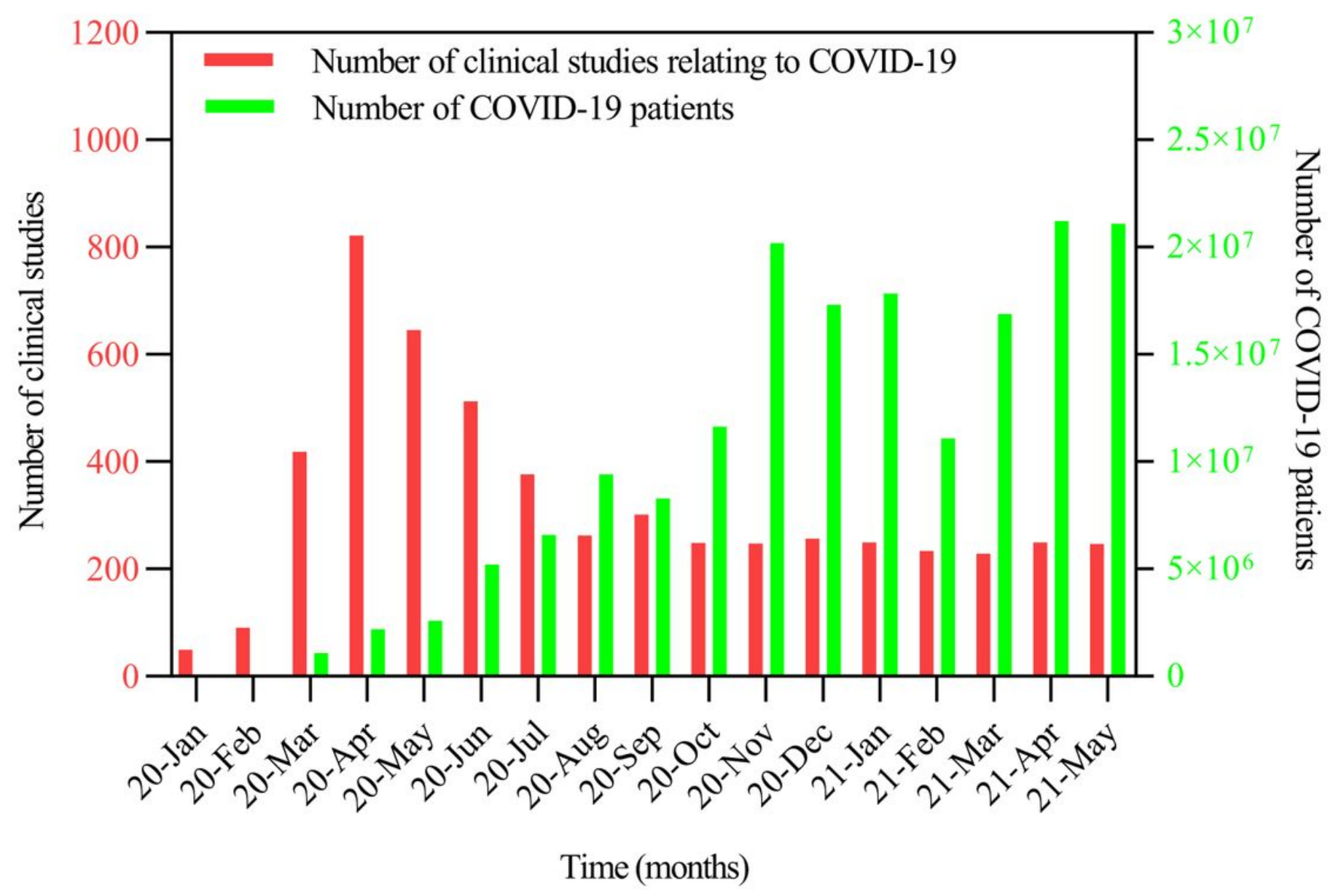

Figure 3

The number of COVID-19 patients and COVID-19-related clinical studies and corresponding time (months) (The red column indicates the number of clinical studies and the green column indicates the number of COVID-19 cases). 

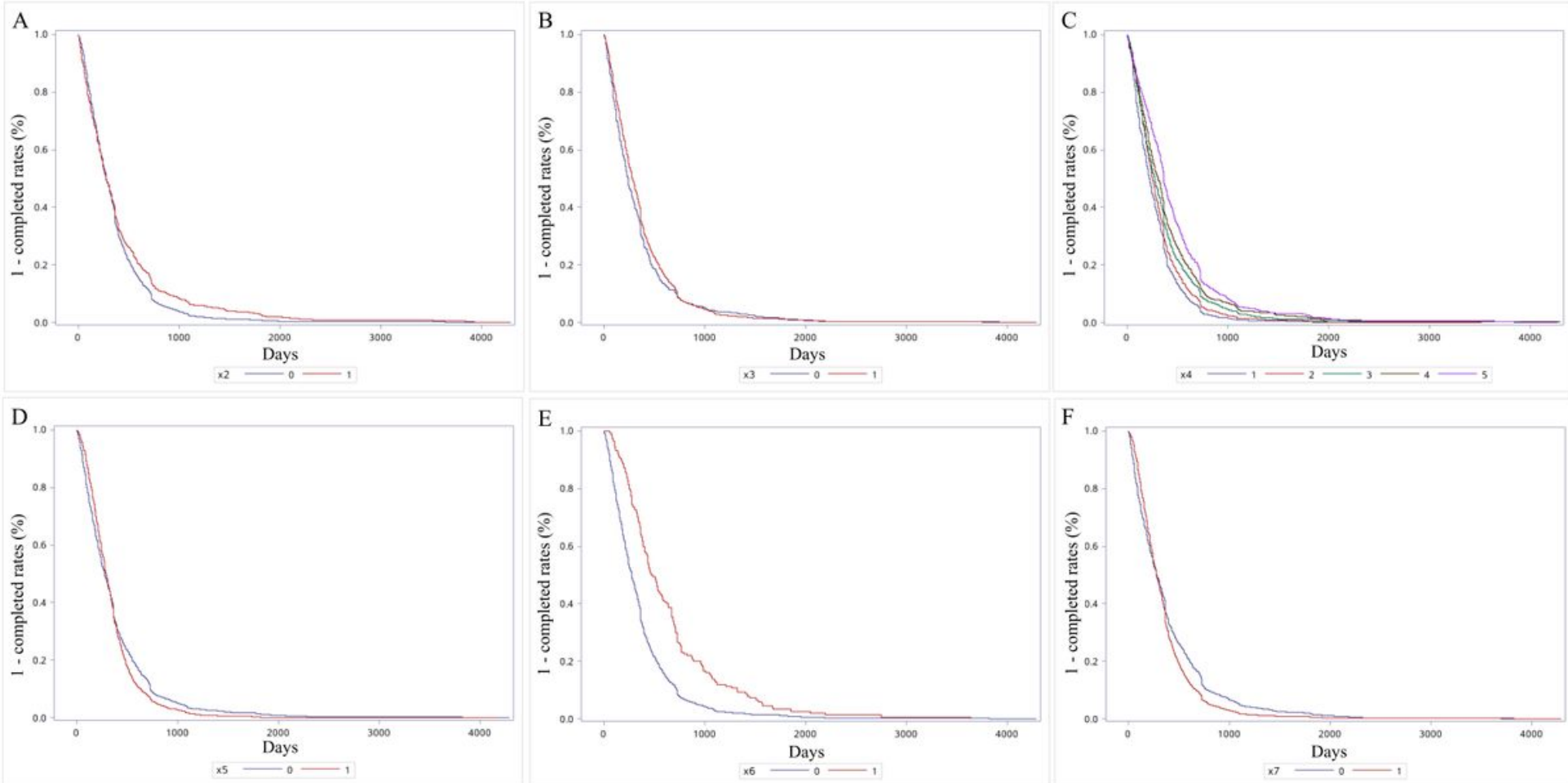

Figure 4

Kaplan-Meier curves for variables in the entire sample. A. $x 2$ indicates Participants including child (0 indicates no and 1indicates yes) (P凶0.01, log-rank test), B. $\times 3$ indicates Participants including older adults ( 0 indicates no and 1 indicates yes) ( $P=0.04$, log-rank test), C. $x 4$ indicates enrollment (1 indicates $\leq 50,2$ indicates $>50$ and $\leq 100,3$ indicates $>100$ and $\leq 300$, 4 indicates $>300$ and $\leq 900$, and 5 indicates $>900$ ) ( $P \bowtie 0.01$, log-rank test), D. $x 5$ indicates funded by industry ( 0 indicates no and 1indicates yes) $(P=0.02$, logrank test), E. $x 6$ indicates funded by $\mathrm{NIH}$ ( 0 indicates no and 1 indicates yes) ( $P=0.02$, log-rank test), and $F$. $x 7$ indicates study type ( 0 indicates observational and 1 indicates interventional) (Pख0.01, log-rank test). 

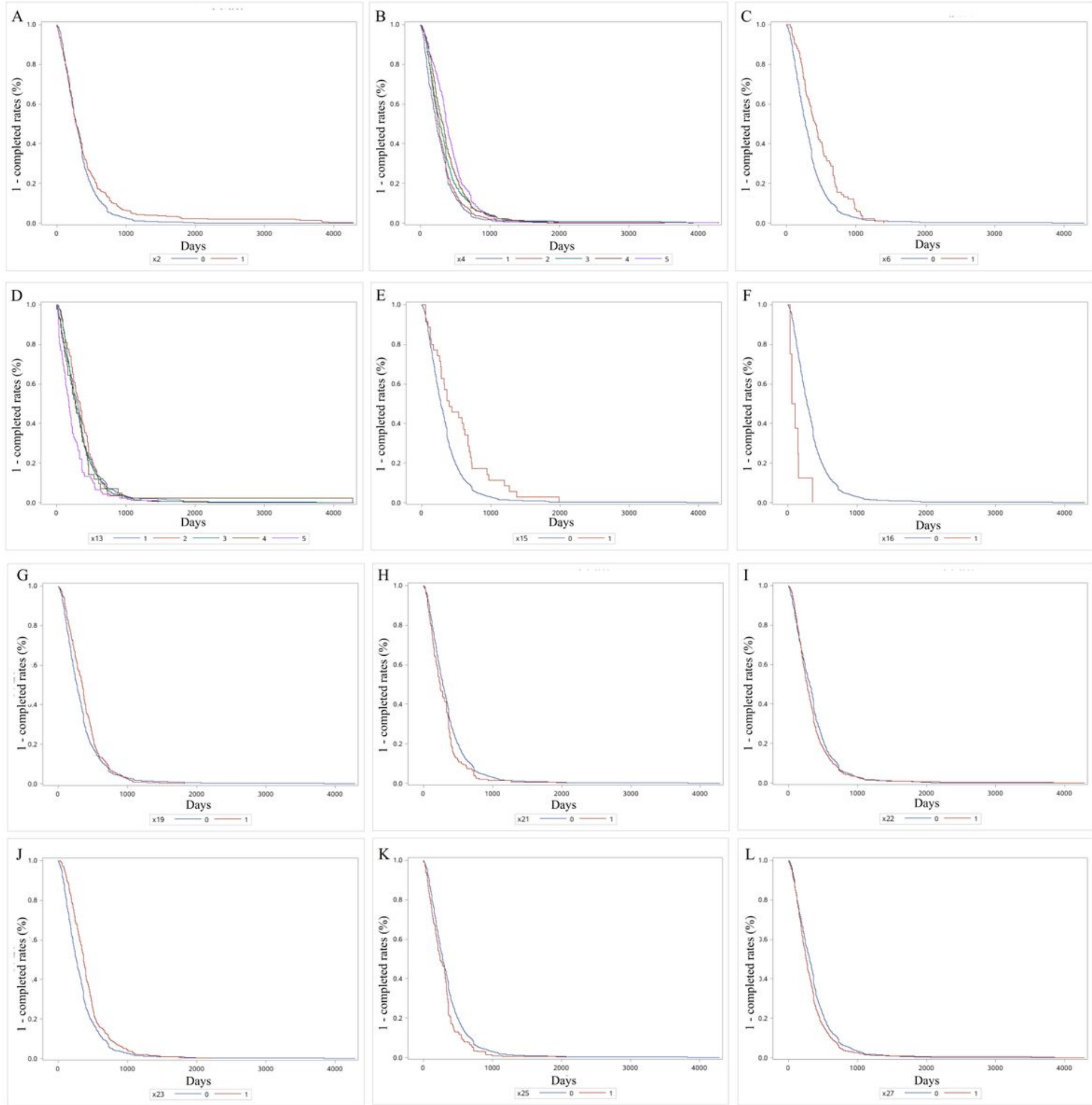

Figure 5

Kaplan-Meier curves for variables in the interventional studies. A. $\times 2$ indicates Participants including child ( 0 indicates no and 1 indicates yes) (Pख0.01, log-rank test), B. $x 4$ indicates enrollment (1 indicates $\leq 50,2$ indicates $>50$ and $\leq 100,3$ indicates $>100$ and $\leq 300,4$ indicates $>300$ and $\leq 900$, and 5 indicates $>900)(P$ $\otimes 0.01$, log-rank test), C. $x 6$ indicates funded by NIH ( 0 indicates no and 1indicates yes) (Pख0.01, log-rank test), D. x13 indicates intervention model (1 indicates Single, 2 indicates sequential, 3 indicates parallel, 4 indicates factorial, and 5 indicates crossover) (Pख0.01, log-rank test), E. x15 indicates basic science (0 
indicates no and 1indicates yes) (Pख0.01, log-rank test), F. x16 indicates device feasibility (0 indicates no and 1 indicates yes) (P凶0.01, log-rank test), G. $\times 19$ indicates prevention ( 0 indicates no and 1 indicates yes) ( $P \otimes 0.01$, log-rank test), H. $x 21$ indicates supportive care ( 0 indicates no and 1 indicates yes) $(P=0.02$, logrank test), I. X22 indicates treatment ( 0 indicates no and 1indicates yes) ( $P=0.02$, log-rank test), G. x23 indicates biological ( 0 indicates no and 1 indicates yes) ( $P \llbracket 0.01$, log-rank test), K. $x 25$ indicates device ( 0 indicates no and 1indicates yes) (Pख0.01, log-rank test), and L. $x 27$ indicates drug ( 0 indicates no and 1 indicates yes) (P凶0.01, log-rank test).
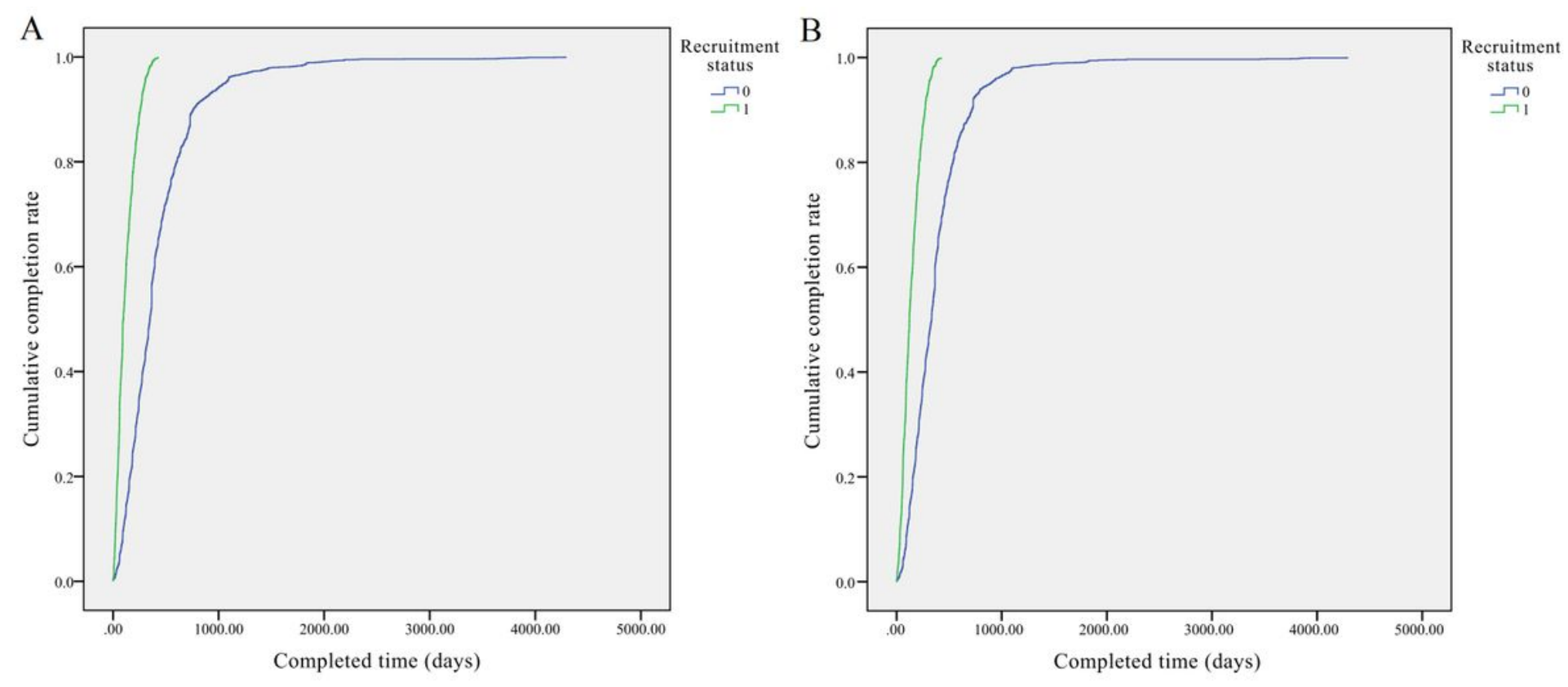

\section{Figure 6}

Cumulative curves for recruitment status in all clinical studies $(A, P<0.01$, log-rank test) and the interventional studies $(B, P<0.01$, log-rank test). 1 indicates completed status and 0 indicates other status. 